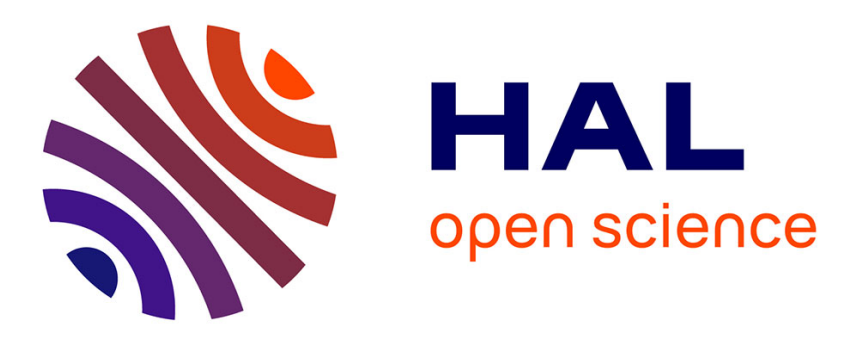

\title{
Model for a sensor inspired by electric fish
}

\author{
Frédéric Boyer, Pol-Bernard Gossiaux, Brahim Jawad, Vincent Lebastard, \\ Mathieu Porez
}

\section{To cite this version:}

Frédéric Boyer, Pol-Bernard Gossiaux, Brahim Jawad, Vincent Lebastard, Mathieu Porez. Model for a sensor inspired by electric fish. IEEE Transactions on Robotics, 2012, 52 (2), pp.492-505. 10.1109/TRO.2011.2175764 . hal-00614081v3

\section{HAL Id: hal-00614081 https://hal.science/hal-00614081v3}

Submitted on 1 Sep 2011

HAL is a multi-disciplinary open access archive for the deposit and dissemination of scientific research documents, whether they are published or not. The documents may come from teaching and research institutions in France or abroad, or from public or private research centers.
L'archive ouverte pluridisciplinaire HAL, est destinée au dépôt et à la diffusion de documents scientifiques de niveau recherche, publiés ou non, émanant des établissements d'enseignement et de recherche français ou étrangers, des laboratoires publics ou privés. 


\title{
Model for a sensor inspired by electric fish
}

\author{
Frédéric Boyer, Pol Bernard Gossiaux, Brahim Jawad, \\ Vincent Lebastard and Mathieu Porez. ${ }^{*} \dagger \xi \S$
}

$1^{\mathrm{er}}$ septembre 2011

\begin{abstract}
Résumé
This article reports the first results from a programme of work aimed at developing a swimming robot equipped with electric sense. After having presented the principles of a bioinspired electric sensor, now working, we will build the models for electrolocation of objects that are suited to this kind of sensor. The produced models are in a compact analytical form in order to be tractable on the onboard computers of the future robot. These models are tested by comparing them with numerical simulations based on the boundary elements method. The results demonstrate the feasibility of the approach and its compatibility with online objects electrolocation, another parallel programme of ours.
\end{abstract}

Electrolocation, active perception, bio-inspired sensor, model reduction, electrokinetics, electrostatics, resistance matrix.

\section{Introduction}

Discovered in the fifties by Lissmann [1], the electric sense or "electrolocation" is used by hundreds of fish species that have co-evolved on both the African and South American continents. This sense is based on the measurement of the perturbations of an electric field fish's emission induced by the environment (see Fig. 1). This sense is ideally suited for navigation in the murky waters of the equatorial forests in which these fishes live. For species of the mormyridae family, electrolocation, whose range is of the order of a body length, is based on the dipolar field created by the polarisation of the body relative to an electric organ discharge (EOD) that is situated just proximal to the tail (Fig. 1). With a higher conductivity than the water, the fish body funnels the emitted field lines like an "electric lens", and makes them cross its electrosensitive skin.

Thanks to a high number of electro-receptors distributed all along its body, the fish's brain processes an instantaneous electric image of its three dimensional environment by a comparison between the transcutaneous currents in the absence of objects and those measured in their presence. Applied to "small objects", for which the applied field is near uniform in the region occupied by the object, typically a sphere of radius $a$ and of center $O$, the principle of the electrolocation can be physically explained by the following equation (see [2]) :

$$
\delta \phi=-a^{3} \chi \frac{\nabla \phi_{0} \cdot \mathbf{r}}{r^{3}}
$$

\footnotetext{
${ }^{*}$ F. Boyer is with the IRCcYN lab, Ecole des Mines de Nantes, France. e-mail : frederic.boyer@mines-nantes.fr.

${ }^{\dagger}$ P.-B. Gossiaux is with the SUBATECH lab, Ecole des Mines de Nantes, France. e-mail : gossiaux@minesnantes.fr.

${ }^{\ddagger}$ B. Jawad is with the IRCcYN lab, CNRS, France. e-mail : brahim.jawad@mines-nantes.fr.

${ }^{\S}$ V. Lebastard is with the IRCcYN lab, Ecole des Mines de Nantes, France. e-mail : vincent.lebastard@minesnantes.fr.

${ }^{\top}$ M. Porez is with the IRCcYN lab, Ecole des Mines de Nantes, France. e-mail : mathieu.porez@mines-nantes.fr.
} 


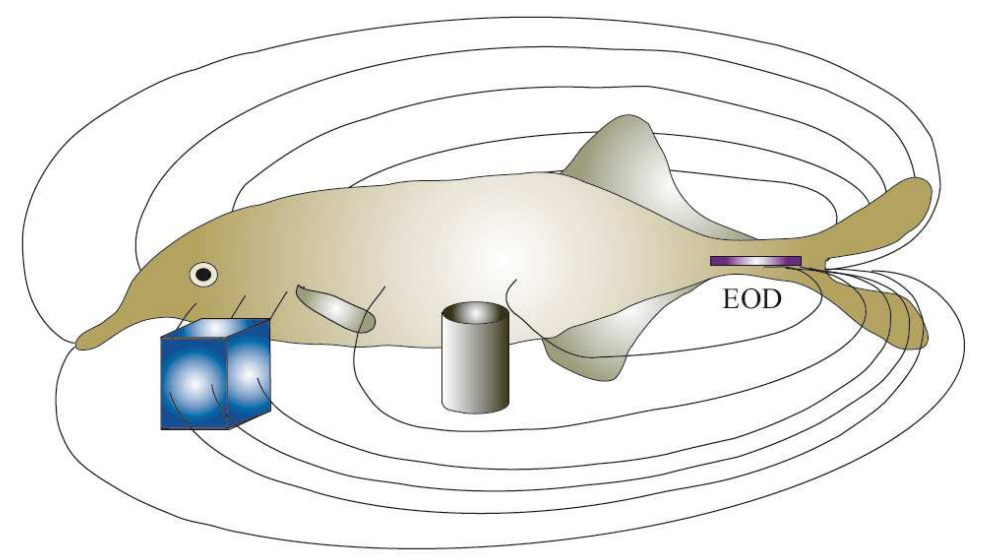

Figure 1 - The electric fish, here the Gnathonemus Petersii. The electric field is distorted by the presence of an object (for instance, an insulating cylinder blocks the field lines whereas a conductive cube funnels them).

where $\delta \mathbf{E}=-\nabla(\delta \phi)$ represents the perturbation at the point $M$ of an exogenous uniform electric field $\mathbf{E}_{0}=-\nabla \phi_{0}$ induced by the sphere centered at $O$, with $\mathbf{r}=\overrightarrow{O M}, r=\|\mathbf{r}\|$ and $\chi$ a "contrast factor" encoding the relative conductivity of the sphere with respect to the water. In such a model, and throughout this article, both the water and the objects are considered as homogenous isotropic ohmic media. It is Rasnow [3] who first applied this perturbation formula (1) to electrolocation. In this context, $\phi_{0}$ models the field produced by the fish, while $\delta \phi$ represents the potential difference with and without the objects, measured at a point on the skin of the fish situated at $\mathbf{r}$ from the object. Intensely researched in biology, this active mode of perception has been little studied in robotics despite its promise for navigation in turbid waters where the high density of particles precludes the use of sonar.

Recently, Mc Iver et al. $[4,5,6,7]$ have exploited this sense in the context of a bio-inspired approach in robotics. They built an experimental set up of four point electrodes placed at the apexes of a rhombus in a rigid moving frame driven by a Cartesian robot [7]. In this system, two electrodes situated at the opposite apexes of the lozenge are polarized in voltage and play the role of the electric organ of discharge while the two opposite electrodes play the role of receivers. This device is a direct implementation of the Rasnow model (1), the generated potential by the two first electrodes being $\phi_{0}$ in (1), while voltage measurement by the receivers is the perturbation $\delta \phi$ induced by the objects placed in the scene. Using the Cartesian motion control of this sensor in a tank, they implemented different electrolocation algorithms for small spheres, demonstrating the feasibility of the principle.

Pursuing similar objectives, we have developed in $[8,9,10,11]$ an alternative technology. Our sensor is a mosaic of electrodes measuring currents, distributed in several connected populations polarized with respect to each other (see Fig. 2 and 3). Hence, in this case, if the electric organ of discharge is still emulated by a voltage control, the measurement is no longer of voltage $(U)$ but of current $(I)$ and we define this method as the $U-I$ mode of measurement to distinguish it from the $U-U$ mode in [7]. One of the contributions of the article is to build, at low cost for the engineer, an analytical model of object electrolocation by a $U-I$ sensor. The model is sufficiently concise and reliable to be applied to real-time navigation of a robot (using observer based algorithms for instance). Furthermore, contrary to (1) which assumes that the electrodes are of insignificant volume, the proposed solution takes into account the fact that every robot, 


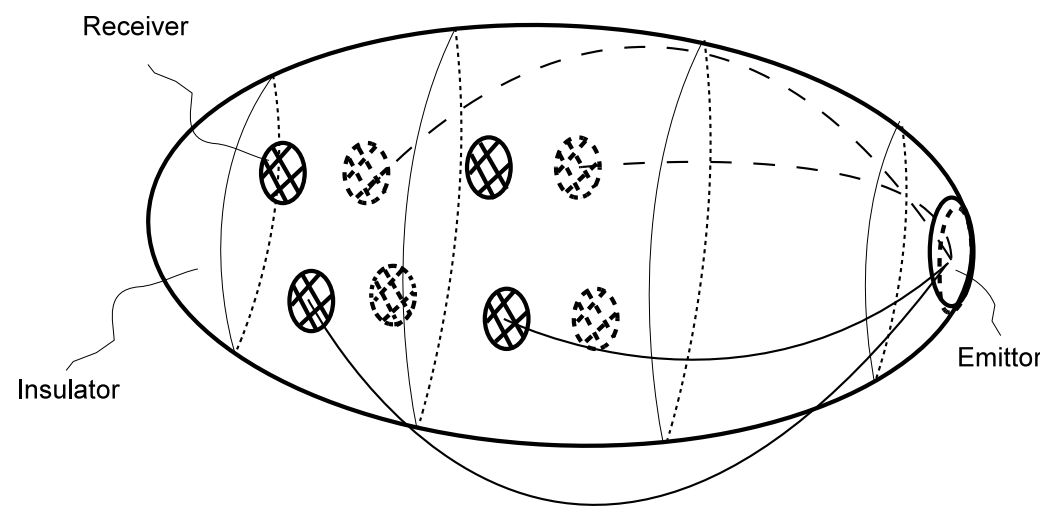

FigURE 2 - Schematic view of the sensor.
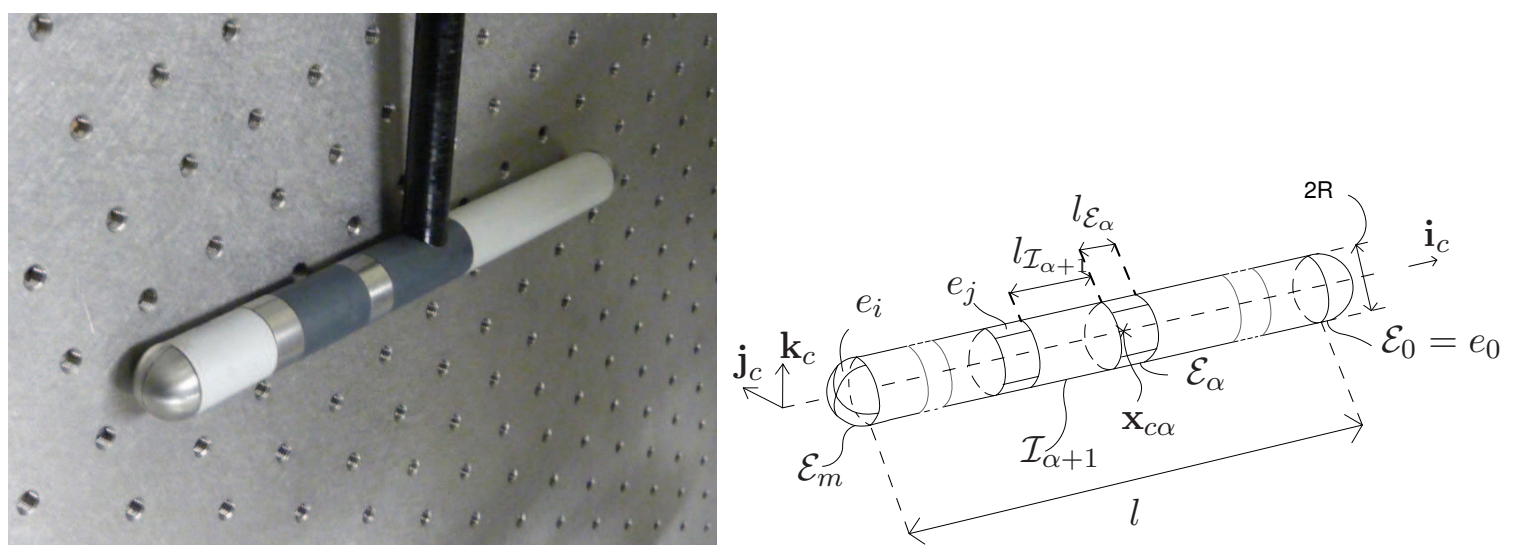

Figure 3 - (top) Picture of a 7-electrode slender sensor organized in 4 polarizable rings, 3 of them being divided in two half rings allowing two lateral (left and right) measurements. (bottom) General schematic view of the slender sensor with its parametrisation.

including the physical volume of the electrodes, will distort the electric fields in a non negligible way. Beyond these practical aspects, the proposed approach introduces some modeling tools not much used by the robotics community, and reveals some fundamental aspects of the electric sense by immersing its modeling in the general framework of the reflection method as it is developed in the field of low numbers hydrodynamics [12]. Once adapted to electrokinetics, this method is well suited for electrolocation. Beyond electric sensing, we believe that it is also well suited for other active senses, such as echo-location based on the sonar technology. Here restricted to the rigid slender vehicles widely used in underwater robotics, the proposed modeling approach aims to be sufficiently generic to represent a first encouraging step towards its future application to the geometry of a fish-like robot. In particular we will see that at the leading order of approximation with respect to small quantities in the problem, the model of the slender sensor working in the mode $U-I$ finally requires two sets of elements. Firstly, an axial conductance matrix and a set of lateral polarization factors that encode the model of measurement in the absence of any object and that can be directly measured on the robot. Secondly, the "reflection lateral matrix" and the "reflection axial matrix" which model the reflection of an incident electric signal projected 
respectively onto the lateral surfaces and to the sensor axis. Finally, beyond a wealth of applications in bio-robotics, the work proposed here could also contribute a valuable reduction scheme for the calculation of the resistance operator of various sensors immersed in conductive media and thus could be applied in other fields.

The article is structured as follows. In section 2, the so-called electric direct problem of electrolocation is stated in a local form. The next section deals with an expansion in successive perturbations based on the reflection method. Then, (section 4) the starting direct electric problem is restated in an integral form which uses Green's identities as does the Boundary Elements Method (BEM). The integral formulation is also applied to the reflections from the sensor to the object when the sensor is considered singly. All these results are applied to derive the approximated models of the emitted signal (from sensor to object (section 5)), reflected signal (from object to sensor (section 6)), and re-reflected signal (from the sensor to the object (section 7)). Section 7 ends with the form of the total currents and their reformulation based on the symmetry properties of the sensor. The model is tested by making comparisons of simulations based on the BEM and the proposed simplified model in section 8. In section 9, the use of the model for electrolocation in robotics is illustrated in a simple experimental test. There is a concluding discussion of the results in section 10 .

\section{Local formulation of the direct problem of electrolocation}

In all the following, for any three dimensional subset $\mathcal{S}$ of $\mathbb{R}^{3}$, we denote by $\partial \mathcal{S}$ its boundary defined as a two dimensional subset of $\mathbb{R}^{3}$, and $\mathcal{S}_{o}=\mathcal{S}-\partial \mathcal{S}$, the set of interior points to $\mathcal{S}$.
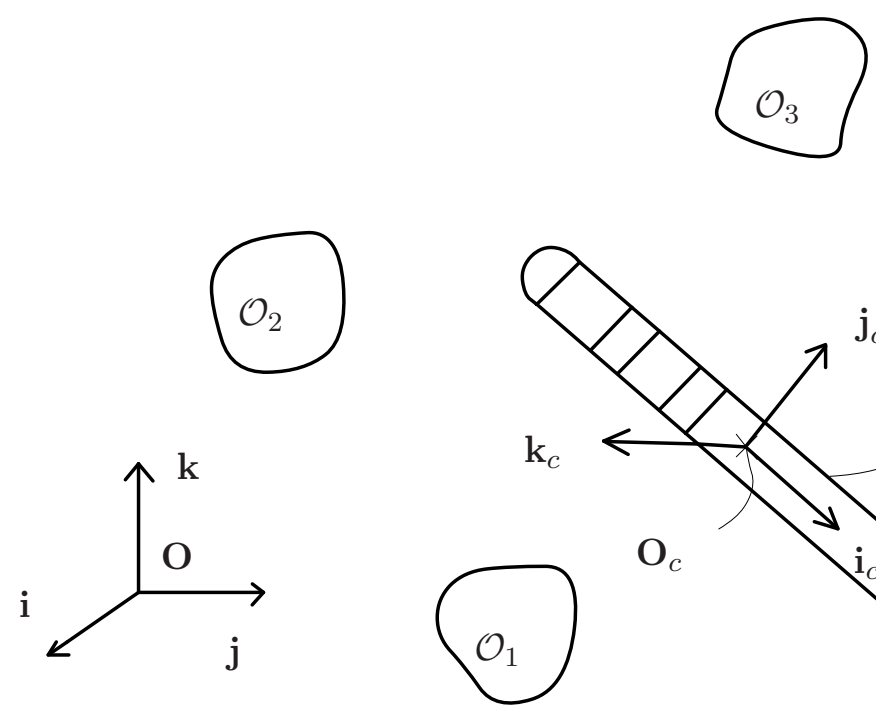

$\mathcal{B}$

FiguRE 4 - Schematic view of the general scene.

Let us consider a set of objects $\mathcal{O}=\cup_{k=1}^{p}\left(\mathcal{O}_{k}\right)$ each one being constituted of an homogenous isotropic Ohmic material of respective conductivities $\gamma_{1,2, . . p}$. These objects are immersed in ordinary water of conductivity $\gamma$. We add an active object denoted $\mathcal{B}$ to the passive objects already present in the scene. This object, which is the sensor, is a mosaic of $n+1$ electrodes defined as ideal conductors whose wet boundaries are denoted $e_{i=0,1, \ldots n}$ (see Fig. 2 and 3). These 
electrodes are gathered into $m+1$ connected populations denoted $\mathcal{E}_{\alpha}, \alpha=0,1,2 \ldots m$ such that $\cup_{i=0}^{i=n} e_{i}=\cup_{\alpha=0}^{\alpha=m} \mathcal{E}_{\alpha}$. In the current design of Fig. 3, these populations are annular and separated from each other by insulating axisymmetric connections $\cup_{\alpha=1}^{\alpha=m} \mathcal{I}_{\alpha}$. On each of the $e_{i}$, we can measure the current that flows across it. On each of the populations $\mathcal{E}_{\alpha}$, we can impose an arbitrary potential except on $\mathcal{E}_{0}$, which plays the role of the origin of potentials, or "mass". For this electrode, we have $\mathcal{E}_{0}=e_{0}$ ( $\mathcal{E}_{0}$ is not divided in electrodes of independent measurement). The obvious reason for setting all electrodes of a given population at the same potential is to avoid the electrical short-circuits that unavoidably otherwise result. The wetted domain of the scene is denoted $\mathcal{D}$. Its boundaries are on the objects (including the sensor) or are infinitely far from them with an outward normal on $\partial \mathcal{D}$ denoted by $\mathbf{n}$. In this context, the equations of physics, which rule the evolution of the measurements for any scene schematized on Fig. 4, can be formulated as the following direct electric problem :

Find the electric potential $\phi$ verifying the following set of equations :

- Laplace equation for the electric potential :

$$
\triangle \phi=0, \forall \mathbf{x} \in \mathcal{D}_{o} .
$$

- Ohm's law in water, with $\mathbf{j}$ the current density field :

$$
\mathbf{j}=-\gamma \nabla \phi, \forall \mathbf{x} \in \mathcal{D}_{o} .
$$

- Conditions on the boundaries of the sensor :

- On the conductors (electrodes) the potential is known (it is imposed) from :

$$
\phi(\mathbf{x})=U_{\alpha}(t), \forall \mathbf{x} \in \mathcal{E}_{\alpha=0,1 . . m},
$$

where $U_{0}(t)=0$, since $\mathcal{E}_{0}$ is the reference electrode which defines the "mass" of the sensor.

- On the insulating connections we have, :

$$
\frac{\partial \phi}{\partial n}(\mathbf{x})=0, \forall \mathbf{x} \in \mathcal{I}_{\alpha=1,2 \ldots m},
$$

where for any function $f, \partial f / \partial n=\nabla f . \mathbf{n}$, the dot denoting the scalar product in $\mathbb{R}^{3}$.

- On the boundaries of the objects (by setting $\mathbf{x}_{ \pm}=\lim _{\varepsilon \rightarrow 0}(\mathbf{x} \pm \varepsilon \mathbf{n})$ ) :

- Conservation of normal currents across the boundaries :

$$
\gamma\left(\frac{\partial \phi}{\partial n}\right)\left(\mathbf{x}_{-}\right)=\gamma_{k}\left(\frac{\partial \phi}{\partial n}\right)\left(\mathbf{x}_{+}\right), \forall \mathbf{x} \in \partial \mathcal{O}_{k=1, \ldots p} .
$$

- Continuity of the potential through the boundaries :

$$
\phi\left(\mathbf{x}_{-}\right)=\phi\left(\mathbf{x}_{+}\right), \forall \mathbf{x} \in \partial \mathcal{O}_{k=1, \ldots p} .
$$

Finally, to complete the formulation of the direct model, the expressions of the measurements must be stated. They can be easily deduced from the electric state of the scene $\phi$, as :

$$
\text { For }: k=0,1,2 \ldots n, I_{k}=-\int_{e_{k}} \mathbf{j} \cdot \mathbf{n} d s=\gamma \int_{e_{k}} \frac{\partial \phi}{\partial n} d s,
$$

where due to the orientation of $\mathbf{n}$, one current is considered positive when it flows out from the sensor and negative when it enters into it. 


\section{Principles for the resolution of the direct problem by the me- thod of successive reflections}

\subsection{Preliminary restrictions and definitions related to the scene}

Before introducing the principle of the method of reflections, we are going to impose some restrictions to the scene. Firstly, from now on we will adopt the sensor design of Fig. 3. In this case, its boundary is composed of a cylinder of radius $R$ and of one hemisphere of radius $R$ at each end. The total length of the sensor is $l$ and its aspect ratio $R / l$ fixes the small quantity with respect to which all the expansions will be achieved. In other terms, the sensor is axisymmetric and slender. As regards the sensor boundaries, the independently polarized populations $\mathcal{E}_{\alpha}$ with $\alpha=1,2 \ldots m-1$, are ring-shaped electrodes, while $\mathcal{E}_{0}$ and $\mathcal{E}_{m}$ refer to the hemi-spherical electrodes at each end. All these electrodes have a length $l_{\mathcal{E}_{\alpha}} \sim R$, and are separated by insulating cylinders $\mathcal{I}_{\beta}(\beta=1,2, \ldots m)$ of radius $R$ and of length $l_{\mathcal{I}_{\beta}}$. Secondly, the set of objects reduces to a unique object $^{1}$ of typical dimension $a$ considered as small relative to the axial dimension of the sensor $l$, i.e. typically of the order of the sensor radius $R$.

\subsection{Presentation of the method with the application to the electrolocation of object}

Despite these simplifications, the exact analytical integration of the direct problem (2-7) is not possible ${ }^{2}$. But because of the strong decrease of the electric interactions with distance, one can approximate the exact solution of the direct problem by a series expansion as follows :

$$
\phi=\phi_{0}+\phi_{1}+\phi_{2}+\ldots=\sum_{i=1}^{\infty} \phi_{i} .
$$

As $i$ increases, $\left|\phi_{i}\right|$ decreases as the inverse of a certain power denoted $s(s$ is a strictly positive integer) of the typical distance separating the sensor and the object, denoted $r$ and which is such that $r>>R$. In practice, for $r \gtrsim 3 R$ the approximation already works, but in order to facilitate the understanding of the subsequent developments we invite the reader to consider that in all the following $r \gtrsim l$. Under these conditions, such an expansion is accessible by resorting to the general perturbation schemes well established in theoretical physics [13]. Here we will follow the method of successive reflections as it is today used in low Reynolds number hydrodynamics for modeling the interactions between particles of a diphasic flow [12]. In the current context, this iterative method is applied as following :

1. Step 0 ("Emission by the sensor") : We ignore the object by removing it from the scene and calculate the response of the sensor which we denote $\phi_{0}$ and which is the solution of the direct problem with no object :

$$
\triangle \phi_{0}=0, \forall \mathbf{x} \in \mathcal{D}_{o} .
$$

Boundary conditions :

$$
\begin{aligned}
\phi_{0}(\mathbf{x}) & =U_{\alpha}(t), \forall \mathbf{x} \in \mathcal{E}_{\alpha=0,1 . . m} \\
\frac{\partial \phi_{0}}{\partial n}(\mathbf{x}) & =0, \forall \mathbf{x} \in \mathcal{I}_{\alpha=1, \ldots m} .
\end{aligned}
$$

1. The multi object case will be discussed later.

2. So that the generally accepted method would be to resort to rather heavy numerical simulation for each configuration of the objects. 
2. Step 1 ("First reflection traveling from the object to the sensor") : $\phi_{0}$ being known from the previous step, we remove the sensor from the scene and calculate $\phi_{1}$ such that the boundary conditions on the object are verified by $\phi_{0}+\phi_{1}$. The system to solve becomes :

$$
\triangle \phi_{1}=0, \forall \mathbf{x} \in \mathcal{D}_{o}
$$

with the boundary conditions on the objects in currents :

$$
\begin{aligned}
\gamma \frac{\partial \phi_{1}}{\partial n}\left(\mathbf{x}_{-}\right) & -\gamma_{k} \frac{\partial \phi_{1}}{\partial n}\left(\mathbf{x}_{+}\right)=\gamma_{k} \frac{\partial \phi_{0}}{\partial n}\left(\mathbf{x}_{+}\right)-\gamma \frac{\partial \phi_{0}}{\partial n}\left(\mathbf{x}_{-}\right) \\
& =\left(\gamma_{k}-\gamma\right) \frac{\partial \phi_{0}}{\partial n}(\mathbf{x}), \quad \forall \mathbf{x} \in \partial \mathcal{O}_{k},
\end{aligned}
$$

and in potential, $\forall \mathbf{x} \in \partial \mathcal{O}$ :

$$
\phi_{1}\left(\mathbf{x}_{-}\right)-\phi_{1}\left(\mathbf{x}_{+}\right)=\phi_{0}\left(\mathbf{x}_{+}\right)-\phi_{0}\left(\mathbf{x}_{-}\right)=0
$$

3. Step 2 ("Second reflection traveling from the sensor to the object") : Again, $\phi_{1}$ is known from step 1 and we remove the object and calculate $\phi_{2}$ such that the boundary conditions on the sensor are verified by $\phi_{0}+\phi_{1}+\phi_{2}$. In other terms, $\phi_{2}$ is solution of the system :

$$
\triangle \phi_{2}=0, \forall \mathbf{x} \in \mathcal{D}_{o},
$$

with the boundary conditions :

$$
\begin{aligned}
\phi_{2}(\mathbf{x}) & =-\phi_{1}(\mathbf{x}), \forall \mathbf{x} \in e_{i=0,1, . . n} \\
\frac{\partial \phi_{2}}{\partial n}(\mathbf{x}) & =-\frac{\partial \phi_{1}}{\partial n}(\mathbf{x}), \forall \mathbf{x} \in \mathcal{I}_{\alpha=1,2 \ldots m} .
\end{aligned}
$$

$\ldots$ and so on...

The principle of this iterative method is summarized in Fig. 5. This perturbation series expansion has a natural physical meaning. Each of the $\phi_{i}$ represents the response to $\phi_{0}+\phi_{1}+$ $\ldots \phi_{i-1}$ alternatively reflected by the object and the sensor through their boundary conditions as the number of reflections $i$ increases (see Fig. 5). This interpretation of the expansion gives its name to the method. We naturally recover in this interpretation the attenuation in $1 / r^{s}$ of the amplitude of the transmitted signals at every traveling between the sensor $\mathcal{B}$ and the object $\mathcal{O}$, with $s$ depending of the size of $\mathcal{B}$ and $\mathcal{O}$. More precisely if we define $\left|\phi_{i+1} / \phi_{i}\right|$ as the attenuation factor introduced by the $i^{\text {th }}$ reflection and measured at some intermediate point located between the sensor and the object we will see that this factor is of the order of $(a / r)^{3}$ if $i$ is even (reflection on the object) or of the order of $(R / r)$ if $i$ is odd (reflection on the sensor). Finally, let us note that these successive reflections have in fact a strong physical meaning, since during very short transient times, the celerity of the signal cannot be assumed to be infinite and the Laplace equation of $\phi$ changes into an actual propagation equation. Thus, in this short time the successive reflections of the method do exist, but very rapidly interfere constructively and destructively in order to generate the steady solution $\phi$. Note also that for the active acoustic sense, or echo-location, these transient reflections are of greater duration. Moreover, in this case, attenuation factors can be decreased by one order since a monopole is physically feasible in acoustics (and in optics) whereas in electrostatics, the emitters being electrically neutral, they can be only dipoles at leading order. Finally, by injecting the potential series expansion (9) into 


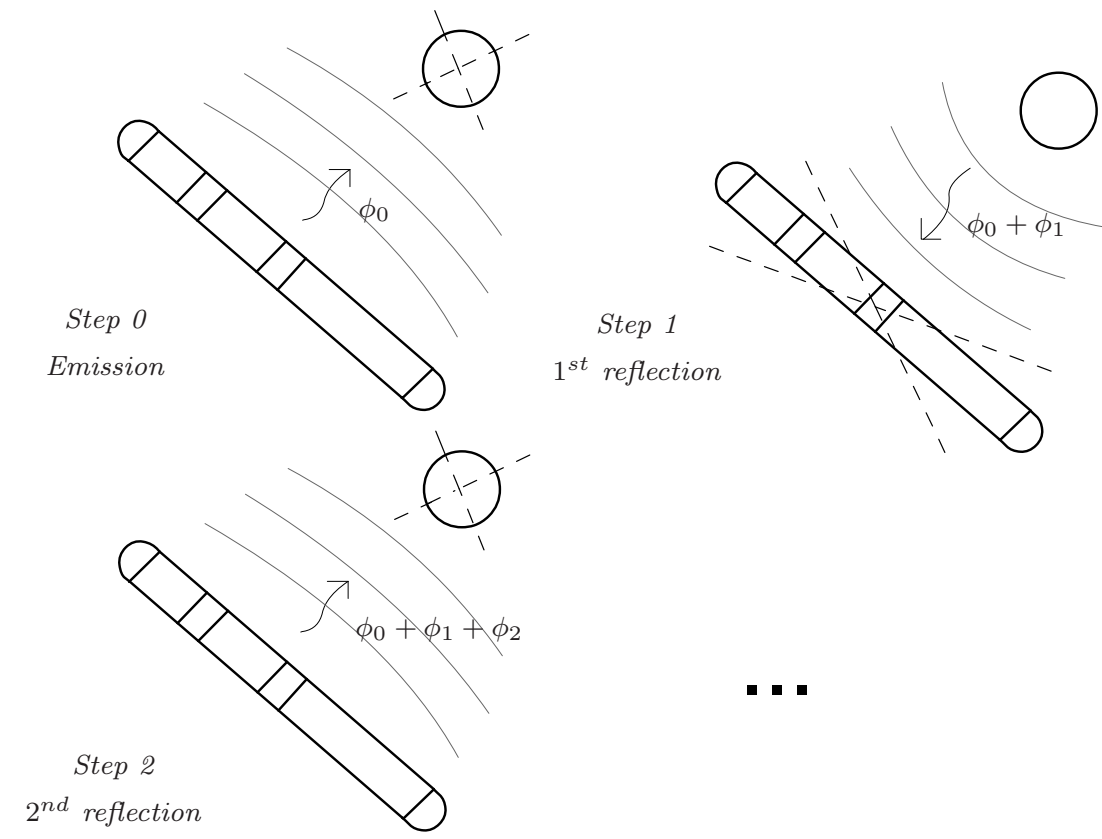

Figure 5 - The method of the successive reflections. First step (top left) : we ignore the object and we solve the Laplace equation for $\phi_{0}$. Second step (top right) : this time we ignore the sensor and we solve the Laplace equation for $\phi_{0}+\phi_{1}$ where $\phi_{1}$ is the perturbation of $\phi_{0}$ induced by the object. Third step (bottom left) : we solve the Laplace equation for $\phi_{0}+\phi_{1}+\phi_{2}$ ignoring the object this time, $\phi_{2}$ being the perturbation of $\phi_{0}+\phi_{1}$. Each novel potential field can be represented as a new contribution (to $\phi$ ) reflected by the object or the sensor. The method can be pursued but as the interactions fall with the inverse of a positive power of the distance, there is no interest in doing so.

the definition of the measured currents (8), every reflection contributes individually to the total measurement which can be expanded as follows :

$$
I \simeq I^{(0)}+I^{(1)}+I^{(2)}+\ldots
$$

where every novel contribution is defined for : $k=0,1,2 \ldots n$, and $i=0,1,2 .$. by :

$$
I_{k}^{(i)}=\int_{e_{k}} \gamma \frac{\partial \phi_{i}}{\partial n} d s
$$

\subsection{Truncation of the expansion : approximation of the second reflection}

Because of the strong attenuation factor introduced by each reflection, we will see that it is reasonable to adopt the following approximation :

$$
\phi \simeq \phi_{0}+\phi_{1}+\phi_{2} .
$$

The measured currents vector $I$ will be consistently approximated by :

$$
I \simeq I^{(0)}+I^{(1)}+I^{(2)} .
$$

Such an approximation is termed in the following the "second reflection approximation". 


\section{Integral formulation of the problem}

By virtue of the Green identities [2], the direct problem (2-7) can be restated in an integral formulation which consists in finding the potential $\phi$ in any interior point of the wetted domain $\left(\mathbf{x} \in \mathcal{D}_{o}\right)$ defined by :

$$
4 \pi \phi(\mathbf{x})=\int_{\partial \mathcal{D}}\left(\frac{1}{r} \frac{\partial \phi}{\partial n}-\phi \frac{\partial(1 / r)}{\partial n}\right) d s
$$

where ${ }^{3} r=\|\mathbf{x}-\mathbf{y}\|, \mathbf{y}$ being an arbitrary point situated on the wetted boundaries of the domain $(\mathbf{y} \in \partial \mathcal{D})$. In particular, if we make any interior point $\mathbf{x}$ tend towards its immediate neighboring point on the boundary (along its normal direction), we find the relation valuable for any $\mathbf{x} \in \partial \mathcal{D}$ :

$$
2 \pi \phi(\mathbf{x})=\int_{\partial \mathcal{D}}\left(\frac{1}{r} \frac{\partial \phi}{\partial n}-\phi \frac{\partial(1 / r)}{\partial n}\right) d s
$$

where we considered that the boundaries are smooth (without any angular point). This last integral equation is at the basis of the numerical BEM [14,15]. In this case, it is required to discretize the boundaries in finite elements and to approximate the potential or current distributions on any element through nodal interpolations. At the end, one obtains an implicit linear differential algebraic system whose dimension corresponds to the number of nodes and whose general form is :

$$
A I+B \Phi=0,
$$

where $I$ and $\Phi$ are respectively the current vector and the potential vector evaluated on the meshing elements, whereas $A$ and $B$ are two matrices which encode the geometry and the conductivity of the medium. In particular if we approximate the potential on each element by a constant value (1-node interpolation), every line of this system represents the contribution of one element to the previous integral equation. Also, since on every element we know either the potential or the current, we have one unknown per equation and the system can be (numerically) solved ${ }^{4}$. Now let us detail further the previous integral equation in the case which interests us. By taking into account the specificities of the problem as it was previously defined, i.e. by separating the sensor's boundaries from those of the objects (i.e. $\partial \mathcal{D}=\partial \mathcal{O} \cup \partial \mathcal{B}$ ), we find :

$$
\begin{aligned}
\forall \mathbf{x} \in \partial \mathcal{B}: & \int_{\partial \mathcal{B}}\left(\frac{1}{r} \frac{\partial \phi}{\partial n}-\phi \frac{\partial(1 / r)}{\partial n}\right) d s+ \\
& \int_{\partial \mathcal{O}}\left(\frac{1}{r} \frac{\partial \phi}{\partial n}-\phi \frac{\partial(1 / r)}{\partial n}\right) d s, \\
\forall \mathbf{y} \in \partial \mathcal{O}:= & \int_{\partial \mathcal{B}}\left(\frac{1}{r} \frac{\partial \phi}{\partial n}-\phi \frac{\partial(1 / r)}{\partial n}\right) d s+ \\
2 \pi \phi(\mathbf{y})= & \int_{\partial \mathcal{O}}\left(\frac{1}{r} \frac{\partial \phi}{\partial n}-\phi \frac{\partial(1 / r)}{\partial n}\right) d s .
\end{aligned}
$$

3. In the following $r$ will denote the distance between any couple of points, one being the current point of the integral and the other being fixed.

4. With $A$ and $B$ defined by $A_{i j}=-\int_{e l t_{i}} \partial\left(1 / r_{i}\right) / \partial n_{j} d s$ and $B_{i j}=(1 / 2) \pi \delta_{i j}+\int_{e l t_{i}}\left(1 / r_{i}\right) d s$, with $\mathbf{x}$ a point running on the $j^{t h}$ element denoted by $e l t_{j}, \mathbf{x}_{i}$ the node location of $e l t_{i}, r_{i}=\left\|\mathbf{x}-\mathbf{x}_{i}\right\|$, and $\mathbf{n}_{j}$ the normal to elt . $_{\text {. }}$ 
From left to right and from above to below we find in the second members of these two equations : the influence of the sensor on itself, the influence of the objects on the sensor, the influence of the sensor on the objects and the influence of the objects on themselves. Finally, (24-25) express the electric equilibrium of the scene.

\subsection{Application to the sensor in the absence of an object}

The previous integral formulation will allow us to calculate the measured currents at each step of the reflection method where we have no object in the scene. Thus, in the second reflection approximation, (24-25) will be used with the boundary conditions of the emission $\phi_{0}$, and the second reflection $\phi_{2}$ respectively. Furthermore, in these two cases we have to remove the object from equation (24) and take into account $\partial \mathcal{B}=\left(\cup_{\alpha=1}^{m} \mathcal{I}_{\alpha}\right) \cup\left(\cup_{\alpha=0}^{m} \mathcal{E}_{\alpha}\right)$ in the resulting equations. Then, it becomes possible to write the following set of integral equations which are verified on each electrode, i.e. $\forall \mathbf{x} \in \mathcal{E}_{\alpha}$ :

$$
\begin{aligned}
2 \pi \phi_{i}(\mathbf{x})= & \sum_{\beta=1}^{m}\left[\int_{\mathcal{I}_{\beta}}\left(\left(\frac{\partial \phi_{i}}{\partial n}\right) \frac{1}{r}-\phi_{i} \frac{\partial(1 / r)}{\partial n}\right) d s\right]+ \\
& \sum_{\beta=0}^{m}\left[\int_{\mathcal{E}_{\beta}}\left(\left(\frac{\partial \phi_{i}}{\partial n}\right) \frac{1}{r}-\phi_{i} \frac{\partial(1 / r)}{\partial n}\right) d s\right] .
\end{aligned}
$$

In the following, we will calculate from (26) the electric responses $I^{(i=0,2)}$ of the sensor. Going further, by injecting in (26) the boundary conditions of $\phi_{0}$ and $\phi_{2}$ given by (11) and (16), we find the two equations which rule the electric equilibrium of the sensor in the step 0 and 2 :

- Emission from the sensor to the object $\left(\phi_{0}\right)$ :

$$
\begin{aligned}
2 \pi U_{\alpha}= & \sum_{\beta=0}^{m}\left[\int_{\mathcal{E}_{\beta}}\left(\frac{\partial \phi_{0}}{\partial n}\right) \frac{d s}{r}-\int_{\mathcal{E}_{\beta}} \frac{\partial(1 / r)}{\partial n} d s U_{\beta}\right] \\
& -\sum_{\beta=1}^{m}\left[\int_{\mathcal{I}_{\beta}} \phi_{0} \frac{\partial(1 / r)}{\partial n} d s\right], \forall \mathbf{x} \in \mathcal{E}_{\alpha} .
\end{aligned}
$$

- Second reflection from the object to the sensor $\left(\phi_{2}\right)$ :

$$
\begin{aligned}
& 2 \pi \phi_{1}(\mathbf{x})=\sum_{\beta=1}^{m}\left[\int_{\mathcal{I}_{\beta}}\left(\frac{\partial \phi_{1}}{\partial n}\right) \frac{d s}{r}+\int_{\mathcal{I}_{\beta}} \phi_{2} \frac{\partial(1 / r)}{\partial n} d s\right] \\
& -\sum_{\beta=0}^{m}\left[\int_{\mathcal{E}_{\beta}}\left(\frac{\partial \phi_{2}}{\partial n}\right) \frac{d s}{r}+\int_{\mathcal{E}_{\beta}} \phi_{1} \frac{\partial(1 / r)}{\partial n} d s\right], \forall \mathbf{x} \in \mathcal{E}_{\alpha} .
\end{aligned}
$$

\section{Model of the emission from the sensor to the object}

The model of emission is set firstly by the model of the measured currents in absence of object in the scene $I^{(0)}$ (section 5.A), and secondly by the model of the ambient field $\phi_{0}$ produced by the inner electric activity of the sensor without object (section 5.B). 


\subsection{Model of the currents $I^{(0)}$}

The goal of this section is to calculate the response of the sensor in terms of the measured currents in the case where we have no object while the sensor is submitted to the boundary conditions of $\phi_{0}$. In the following this problem is solved by the BEM. This approach has the advantage of not requiring any approximation of the geometry of the sensor while the heavy computations it requires can be done once for all for a given sensor. By meshing the electrodes $\mathcal{E}_{\alpha}$ and the insulating surfaces $\mathcal{I}_{\alpha}$ by respectively $n_{c}$ and $n_{i}$ elements, the general linear implicit system (23) can be detailed as :

$$
\begin{array}{r}
\left(\begin{array}{cc}
A_{c c} & A_{c i} \\
A_{i c} & A_{i i}
\end{array}\right)\left(\begin{array}{c}
I_{c}^{(0)} \\
0
\end{array}\right)+\left(\begin{array}{cc}
B_{c c} & B_{c i} \\
B_{i c} & B_{i i}
\end{array}\right) \\
=\left(\begin{array}{c}
\Phi_{c}^{(0)} \\
\Phi_{i}^{(0)}
\end{array}\right) \\
=\left(\begin{array}{c}
0_{n_{c}} \\
0_{n_{i}}
\end{array}\right)
\end{array}
$$

where $I_{c}^{(0)}, \Phi_{c}^{(0)}$ and $\Phi_{i}^{(0)}$ represent respectively the vectors of currents and potentials on the boundaries of the conductors and the insulators in absence of any exterior object. Then we define the $(n+1) \times 1$ vector of measured currents as well as the $(m+1) \times 1$ vector of the potentials imposed to the electrodes $\mathcal{E}_{\alpha}$ :

$$
\Phi_{c}^{(0)}=P_{n_{c}, m+1} U, I^{(0)}=P_{n_{c}, n+1}^{T} I_{c}^{(0)},
$$

where $P_{n_{c}, m+1}$ distributes the vector of the $m+1$ imposed potentials on the $n_{c}$ elements which mesh the borders of the electrodes $\mathcal{E}_{\alpha}$, and $P_{n_{c}, n+1}^{T}$ performs the summation of the $n_{c}$ currents of the elements which compose the electrodes $e_{i}$ to produce the vector of the $n+1$ independent measured currents. Then if we use the second line of (29) to express $\Phi_{i}$ as a function of $I_{c}^{(0)}$ and $\Phi_{c}^{(0)}$ which we then re-inject in the first line of (29), we obtain the reduced implicit linear system :

$$
\widetilde{A}_{c c} I_{c}^{(0)}+\widetilde{B}_{c c} \Phi_{c}^{(0)}=0_{c}
$$

where : $\widetilde{A}_{c c}=A_{c c}-B_{c i} B_{i i}^{-1} A_{i c}$ and $\widetilde{B}_{c c}=B_{c c}-B_{c i} B_{i i}^{-1} B_{i c}$ are two squared matrices. Finally by using the two projectors (30), we obtain the model of the measured currents :

$$
I^{(0)}=C^{(0)} U
$$

where we have introduced the $(n+1) \times(m+1)$ conductance matrix in the absence of any object :

$$
C^{(0)}=\lim _{\left(n_{c}, n_{i}\right) \rightarrow \infty}-P_{n_{c}, n+1}^{T} \widetilde{A}_{c c}^{-1} \widetilde{B}_{c c} P_{n_{c}, m+1},
$$

which can be off-line computed once for all, or alternatively deduced from a preliminary calibration of the sensor, far from any object. In the following we will also use the $(m+1) \times(m+1)$ square axial conductance matrix defined by $\bar{C}^{(0)}=P_{+} C^{(0)}$ where $P_{+}=P_{n+1, m+1}^{T}$ projects the currents crossing the electrodes $e_{i}$ onto those crossing the electrodes $\mathcal{E}_{\alpha}$ (by performing a summation of the currents ring by ring, which is meant by the index "+") according to :

$$
\bar{I}^{(0)}=P_{+} I^{(0)} .
$$

In $(34), \bar{I}^{(0)}$ refers to the reduced vector of axial currents with no object. To illustrate these computations, let us apply them to the sensor presented in the picture (see Fig. 3) for which the electrodes $\mathcal{E}_{\alpha}$ are spliced into two opposite (left/right) half rings and whose dimensions are those of section VIII. Then, for $n+1=7$ and $m+1=4$ and with a meshing of 2444 1-node triangular 
elements, the previous computations give the following axial conductance matrix in the absence of any object :

$$
\bar{C}^{(0)} \simeq \frac{\gamma}{100}\left(\begin{array}{cccc}
7.6534 & -3.1370 & -2.3053 & -2.1829 \\
-3.1370 & 8.3933 & -3.2027 & -2.0804 \\
-2.3053 & -3.2027 & 7.8032 & -2.3190 \\
-2.1829 & -2.0804 & -2.3190 & 6.6052
\end{array}\right) .
$$

Finally, let us notice that by replacing (32) by the reduced (axial) relation :

$$
\bar{I}^{(0)}=\bar{C}^{(0)} U,
$$

we lose no information if we complete (35) with the distribution relation :

$$
I^{(0)}=D_{+} \bar{I}^{(0)}=D_{+} P_{+} I^{(0)},
$$

where $D_{+}$is a $(n+1) \times(m+1)$ matrix said of distribution since in the general case, $D_{+}$is defined by : $D_{+i \alpha}=I_{i}^{(0)} / \bar{I}_{\alpha}^{(0)}$, if $e_{i} \subset \mathcal{E}_{\alpha}$, and $D_{+i \alpha}=0$, otherwise. Finally, when the electrodes $\mathcal{E}_{\alpha}$ are partitioned along the sensor axis into the electrodes $e_{i}$ (as on the example of Fig. 3), then due to the sensor axisymmetry, $D_{+i \alpha}=A_{i} / A_{\alpha}$ where $A_{i}$ and $A_{\alpha}$ are the areas of $e_{i}$ and $\mathcal{E}_{\alpha}$ respectively.

\subsection{Model of $\phi_{0}$, model of the applied field $\mathrm{E}_{0}$}

The potential field generated by the electric activity of the sensor in the absence of any object is simply defined by removing the influence of the objects in the integral equation (24), which gives :

$$
\begin{array}{r}
4 \pi \phi_{0}(\mathbf{y})=\sum_{\alpha=0}^{m} \int_{\mathcal{E}_{\alpha}}\left(\frac{\partial \phi_{0}}{\partial n}\right) \frac{1}{r} d s \\
-\sum_{\alpha=0}^{m}\left(\int_{\mathcal{E}_{\alpha}} \frac{\partial\left(1 / r_{\alpha}\right)}{\partial n} d s\right) U_{\alpha}-\sum_{\alpha=1}^{m} \int_{\mathcal{I}_{\alpha}} \phi_{0} \frac{\partial(1 / r)}{\partial n} d s,
\end{array}
$$

where $\mathbf{y}$ refers now to any point of the domain (without object) exterior to the sensor, and $\mathbf{n}$ now points toward the water. By performing a perturbation series expansion of (37) with respect to the small quantity $R / r \simeq R / l$, we can show (see Appendix A for more details) that:

$$
\phi_{0}(\mathbf{y})=\frac{1}{4 \pi} \sum_{\alpha=0}^{m} \frac{\bar{I}_{\alpha}^{(0)}}{\gamma r_{\alpha}}+O\left(\frac{R}{r}\right) .
$$

Thus, the electric field applied by the sensor in any point of the space and in particular at the point $\mathbf{y}_{c}$ which is the center of the object $\mathcal{O}$, can be written at the leading order in $R / r$ :

$$
\mathbf{E}_{0}\left(\mathbf{y}_{c}\right)=\frac{-1}{4 \gamma \pi} \sum_{\alpha=0}^{m}\left(\nabla_{\mathbf{y}} \frac{1}{r}\right) \bar{I}_{\alpha}^{(0)}=\frac{1}{4 \gamma \pi} \sum_{\alpha=0}^{m} \frac{\bar{I}_{\alpha}^{(0)} \mathbf{r}_{\alpha}}{\left\|\mathbf{r}_{\alpha}\right\|^{3}},
$$

where each $\mathbf{r}_{\alpha}$ refers to the position vector of $\mathbf{y}_{c}$ with respect to the center of the conducting ring $\mathcal{E}_{\alpha}$. Finally, let us notice that the applied field (39) is nothing but that which would be produced by a distribution of punctual charges $\rho(\mathbf{x})=\sum_{\alpha=0}^{m} q_{\alpha} \delta\left(\mathbf{x}-\mathbf{x}_{c \alpha}\right)$ located at the centers of the electrodes $\mathbf{x}_{c \alpha}$ and of equivalent intensities $q_{\alpha}=\epsilon \bar{I}_{\alpha}^{(0)} / \gamma$ where $\epsilon$ is the electrical permittivity of the water. One should note that this result is not so trivial as it may appear at first view, since other physical charges are also present on the sensor (located on the insulating cylinders for instance, or on the internal faces of its electrodes). It is precisely the purpose of Appendix A to demonstrate that those charges do not contribute at leading order due to the slender geometry of the sensor. 


\section{Model of the first reflection (from the object to the sensor)}

This model requires the calculation of the potential $\phi_{1}$ reflected by the perturbative object (section 6.1), here supposed to be reduced to a unique object $\mathcal{O}$ and to the vector of currents $I^{(1)}$ produced by $\phi_{1}$ (section 6.2).

\subsection{Model of $\phi_{1}$, calculation of the response of a small object introduced into an external field $\mathrm{E}_{0}$}

To calculate $\phi_{1}$, one can consider directly the partial differential equations which rule it, and in particular the boundary conditions (13-14) into which we inject the general form of the solutions to the Laplace equations expanded in a basis of spherical harmonics. Regarding the second member of (13-14), we suppose that the object is small enough to reasonably approximate $\mathbf{E}_{0}$ in the whole domain of the object by its value in its center $\mathbf{y}=\mathbf{y}_{c}$, given by (39). Finally, a term by term identification process on the basis of the spherical harmonics gives the following electric response of the object to the external field :

$$
\phi=\phi_{0}+\phi_{1}=-\mathbf{E}_{0} \cdot \mathbf{r}+\frac{\mathbf{p}\left(\mathbf{E}_{0}\right) \cdot \mathbf{r}}{r^{3}}
$$

where $\mathbf{p}$ is the dipolar moment vector of the object $\mathcal{O}$ induced by the application of the external field $\mathbf{E}_{0}$. In the case of two Ohmic materials (one constituting the small object, the other being water), its response is totally encoded into the linear relation :

$$
\mathbf{p}\left(\mathbf{E}_{0}\right)=\mathbf{P} . \mathbf{E}_{0},
$$

where $\mathbf{P}$ is the so-called two order tensor of polarizability of the object (here $\mathcal{O}$ ). It encodes both the geometry of the object and the electric properties of its material with respect to those of its surroundings. For instance, in the case of a sphere of small size, the isotropic geometry of the object does not privilege any polarization direction and we simply have :

$$
\mathbf{P}=\chi a^{3} \mathbf{1},
$$

where $\chi=\left(\gamma_{1}-\gamma\right) /\left(2 \gamma-\gamma_{1}\right)$ is the contrast factor of the two materials composing the scene, i.e. the water of conductivity $\gamma$ and the object $\mathcal{O}$ of conductivity $\gamma_{1}, a$ is the radius of the sphere and $\mathbf{1}$ is the Kronecker tensor.

\subsection{Model of the currents $I^{(1)}$}

In this section, $\mathbf{n}$ refers to the outward normal vector on the sensor. Also, the currents $I^{(1)}$ can be deduced from the integral relations (8) after one has changed its sign :

$$
I_{k}^{(1)}=-\gamma \int_{e_{k}} \frac{\partial \phi_{1}}{\partial n} d s .
$$

which represents the geometric flux of the electric field reflected by the object. Finally, a calculation based on the perturbations series expansion of (43) with respect to the lateral dimensions of the sensor (detailed in Appendix B) gives at the leading order where the first reflection field is approximately uniform on the $\mathcal{E}_{\alpha}$ :

$$
I^{(1)} \simeq L \bar{I}^{(0)}=L \bar{C}^{(0)} U
$$

where we have introduced the lateral reflection matrix :

$$
L_{k \beta}=-\frac{\bar{A}_{k}}{4 \pi r_{\alpha(k)}^{3}}\left(2 c \nu_{k} \mathbf{e}_{r \alpha(k)}+c \mu_{k} \mathbf{e}_{\theta \alpha(k)}\right) \cdot \mathbf{P} \cdot\left(\frac{\mathbf{r}_{\beta}}{r_{\beta}^{3}}\right),
$$


$\alpha(k)$ being the index of the ring $\mathcal{E}_{\alpha}$ to which $e_{k}$ belongs, while all other parameters of (45) are represented in Fig. 6 and introduced along the calculations of the Appendix B.

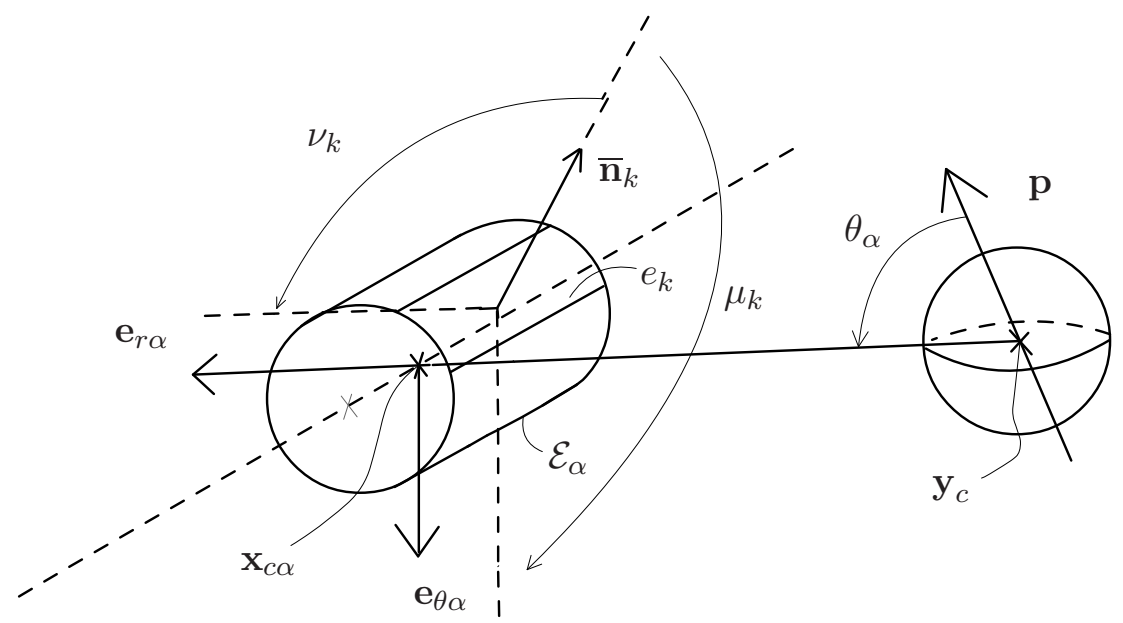

Figure 6 - The perturbation $I^{(1)}$. A schematic view of one perturbated $\mathcal{E}_{\alpha}$ of the sensor is depicted. The perturbation $I^{(1)}$ gives lateral information by measuring the net flux of $\gamma \mathbf{E}_{1}$ across the electrodes $e_{i}$. Like the fish, the sensor is able to know which "side" of its body is more affected by the presence of the object.

Starting from (45) it is useful to split the model of the currents $I^{(1)}$ into two sub models : the axial model (denoted $I_{a x}^{(1)}$ ) and the lateral model (denoted $I_{\text {lat }}^{(1)}$ ), respectively associated with the flow of $\gamma \mathbf{E}_{1}$ entering in parallel and perpendicularly to the axis of the sensor. To do this, we can rewrite $\mathbf{n}$ in (43) as $\mathbf{n}=s \lambda \mathbf{e}_{\rho}+c \lambda \mathbf{e}_{X}=\mathbf{n}_{\perp}+\mathbf{n}_{\|}$, where we have introduced the cylindrical coordinates $(\rho, \psi, X)$ of the sensor such that $\mathbf{i}_{c}=\mathbf{e}_{X}$, and where $\lambda(X)$ is the deviation angle relative to the axis of the sensor of all the normal vectors to the surface of a lateral strip of length $d X$ centered in $X$. It then remains to re-apply the previous computations to obtain the model $I_{l a t}^{(1)}$ and $I_{a x}^{(1)}$ in the matrix form (45), i.e. :

$$
I_{a x}^{(1)} \simeq L_{a x} \bar{C}^{(0)} U, \quad I_{l a t}^{(1)} \simeq L_{l a t} \bar{C}^{(0)} U,
$$

where we have introduced the reflection axial matrix and the reflection lateral matrix, which can be detailed as :

$$
L_{a x, k \beta}=\frac{-\bar{A}_{k \|}}{4 \pi r_{\alpha(k)}^{3}}\left(2 c \nu_{k \|} \mathbf{e}_{r \alpha(k)}+c \mu_{k \|} \mathbf{e}_{\theta \alpha(k)}\right) . \mathbf{P} \cdot\left(\frac{\mathbf{r}_{\beta}}{r_{\beta}^{3}}\right),
$$

as well as :

$$
L_{l a t, k \beta}=\frac{-\bar{A}_{k \perp}}{4 \pi r_{\alpha(k)}^{3}}\left(2 c \nu_{k \perp} \mathbf{e}_{r \alpha(k)}+c \mu_{k \perp} \mathbf{e}_{\theta \alpha(k)}\right) . \mathbf{P} .\left(\frac{\mathbf{r}_{\beta}}{r_{\beta}^{3}}\right),
$$

with $c \nu_{k \perp}=\mathbf{e}_{r \alpha} \cdot \overline{\mathbf{n}}_{k \perp}, c \mu_{k \perp}=\mathbf{e}_{\theta \alpha} \cdot \overline{\mathbf{n}}_{k \perp}, c \nu_{k \|}=\mathbf{e}_{r \alpha} \cdot \overline{\mathbf{n}}_{k \|}, c \mu_{k \|}=\mathbf{e}_{\theta \alpha} \cdot \overline{\mathbf{n}}_{k \|}$, as well as $\bar{A}_{k \|} \overline{\mathbf{n}}_{k \|}=$ $\int_{e_{k}} c \lambda \mathbf{e}_{X} d s$, and : $\bar{A}_{k \perp} \overline{\mathbf{n}}_{k \perp}=\int_{e_{k}} s \lambda \mathbf{e}_{\rho} d s$. In the next section, we will establish that $I_{a x}^{(1)}$ is a sub-dominant contribution of the total axial current, while $I_{\text {lat }}^{(1)}$ is a dominant contribution to the so-called lateral currents that permits the resolution of the azimuthal ambiguity of the object position. 


\section{Second reflection (from the sensor to the object), the full model in the approximation of the second reflection}

\section{$7.1 \quad$ Model of $I^{(2)}$}

The goal consists here in calculating the currents produced by the second reflection by the sensor $\left(\phi_{2}\right)$, i.e. the component $I^{(2)}$ of $I$ (total). For that purpose, we have to reconsider the integral equations (28) which encode the response (in terms of currents) of the sensor to the polarization imposed by the reflected potential $\phi_{1}$. We look for this response under the form $I^{(2)}=I_{a x}^{(2)}+I_{l a t}^{(2)}$ where the first component is due to the polarization along the sensor axis (i.e. due to the differences of $\phi_{1}$ along the sensor axis), while the second component represents the response of the sensor to the lateral polarization (i.e. the gradient of $\phi_{1}$ perpendicular to the sensor axis).

\subsubsection{Model of $I_{a x}^{(2)}$}

Keeping the leading order of a perturbation expansion of (28) with respect to the lateral dimensions of the sensor (see Appendix $\mathrm{C}$ ) shows that the first integral contribution due to the insulating boundaries is negligible while on each $\mathcal{E}_{\alpha}, \phi_{1}$ can be approximated as being uniform and equal to $\phi_{1}\left(\mathbf{x}_{c \alpha}\right)$. Thus, at the leading order, (28) takes the same expression as (27), with $\phi_{2}$ replacing $\phi_{0}$ and for $\alpha=0,1, \cdots, m,-\phi_{1}\left(\mathbf{x}_{c \alpha}\right)$ replacing $U_{\alpha}$. Hence, at the leading order the calculation of $\bar{I}^{(2)}=P_{+} I^{(2)}$ can be simply deduced from the model of $\bar{I}^{(0)}$, by replacing $U=\left(0, U_{1}, U_{2}, \ldots U_{m}\right)^{T}$ in (35) by the vector $-\Phi_{1}=-\left(\Phi_{1,0}, \Phi_{1,1}, \ldots \Phi_{1, m}\right)^{T}$ defined by :

$$
\text { For } \alpha=0,1 \ldots m: \Phi_{1, \alpha}=\phi_{1}\left(\mathbf{x}_{c \alpha}\right) \text {, }
$$

which yields :

$$
\bar{I}^{(2)}=-\bar{C}^{(0)} \Phi_{1} .
$$

Then, if we refer to the expression of $\phi_{1}$ given by (40) in which we first inject the expression (39) of $\mathbf{E}_{0}$, we find, with all position vectors pointing from the sensor to the object:

$$
\Phi_{1 \alpha}=\frac{\mathbf{r}_{\alpha} \cdot \mathbf{P} \cdot \mathbf{E}_{0}}{\left\|\mathbf{r}_{\alpha}\right\|^{3}}=-\frac{1}{4 \pi \gamma} \sum_{\beta=0}^{m}\left(\frac{\mathbf{r}_{\alpha} \cdot \mathbf{P} \cdot \mathbf{r}_{\beta}}{\left\|\mathbf{r}_{\alpha}\right\|^{3}\left\|\mathbf{r}_{\beta}\right\|^{3}}\right) \bar{I}_{\beta}^{(0)},
$$

which can be rewritten in the matrix form :

$$
\Phi_{1}=K \bar{I}^{(0)}
$$

where $K$ is a matrix whose components can be detailed as :

$$
K_{\alpha \beta}=-\frac{1}{4 \pi \gamma} \frac{\mathbf{r}_{\alpha} \cdot \mathbf{P} \cdot \mathbf{r}_{\beta}}{\left\|\mathbf{r}_{\alpha}\right\|^{3}\left\|\mathbf{r}_{\beta}\right\|^{3}} .
$$

In the following $K$ is named the axial reflection matrix since it encodes how the potentials reflected by the object are applied onto the electrodes $\mathcal{E}_{\alpha}$ aligned along the sensor axis. Finally, these reflected potentials will then create a reaction of the currents of the sensor, once again modeled by the axial conductance matrix in the absence of any object :

$$
\bar{I}^{(2)}=-\bar{C}^{(0)} K \bar{I}^{(0)}=-\bar{C}^{(0)} K \bar{C}^{(0)} U .
$$

This simple relation, which requires no additional knowledge of the sensor, is a virtue of its slenderness (see Appendix C for more details). Finally, remarking that (36) can be prolonged to the case of any axisymmetric boundary conditions applied to the sensor with no object, we have at the leading order of approximation : $I_{a x}^{(2)}=D_{+} \bar{I}^{(2)}$. 


\subsubsection{Model of $I_{\text {lat }}^{(2)}$}

Next, pushing the expansion of (28) with respect to the lateral dimensions at the next order (see Appendix D), allows one to capture the lateral response $I_{\text {lat }}^{(2)}$ such that $I^{(2)}=I_{a x}^{(2)}+I_{\text {lat }}^{(2)}$. In fact, from such an expansion, it is easy to show that the electric field of the second reflection is related to that of the first reflection through simple linear relations, which once inserted into (17) and based on considerations exploiting the axisymmetry of the sensor give:

$$
I_{\text {lat }}^{(2)}=S_{\perp} I_{\text {lat }}^{(1)}
$$

In (55), $S_{\perp}$ is a diagonal matrix whose components, denoted by $s_{\perp \alpha(k)}$, are positive and identical for all the electrodes $e_{k}$ of the same $\mathcal{E}_{\alpha}$. From a physical point of view, the $S_{\perp}$ matrix models the reinforcement of the current $I_{k}^{(1)}$ by the lateral polarization of $\mathcal{E}_{\alpha(k)}$ superimposed to the funneling effect of the insulating boundaries neighboring $\mathcal{E}_{\alpha(k)}$. In section 8, we will see how it is possible to directly measure the $S_{\perp}$ matrix through a preliminary calibration.

\subsection{Approximation of the second reflection, axial-lateral decomposition of the currents}

\subsubsection{Justification of the second reflection approximation}

We have now at our disposal all the results required to justify the second reflection approximation (19) and (20). As regards the potentials, combining (39) and (36), and because $\bar{C}^{(0)} \gamma^{-1}=O(R)$ (which can be easily shown by expanding (27) with respect to the lateral dimensions of the sensor), it appears that, reflection after reflection, when the signal passes the sensor it decreases by a factor $R / r$ (i.e. of one order of magnitude). On the other hand, examining (41-43) shows that when the signal passes in transit by the object, it is attenuated by a factor of order $(R / r)^{3}$ (i.e. of 3 orders). Also, we have $\left|\phi_{0} / U\right|=\left|\phi_{2} / \phi_{1}\right|=O(R / r)$ while $\left|\phi_{1} / \phi_{0}\right|=O\left((R / r)^{3}\right)$. Inspecting the currents is even more simple since it suffices to consider (45) and (55) to find, with from (46), (54) $L=O\left((R / r)^{5}\right)$ and $\gamma K=O\left(R^{3} / r^{4}\right)$ : $\left|I^{(1)} / I^{(0)}\right|=O\left((R / r)^{5}\right)$ and $\left|I^{(2)} / I^{(0)}\right|=O\left((R / r)^{4}\right)$. Finally, pushing the method to the next reflection would add contributions such that $\left|I^{(4)} / I^{(0)}\right|=O\left((R / r)^{8}\right)$ and $\left|I^{(3)} / I^{(0)}\right|=O\left((R / r)^{10}\right)$. Compared to the neglected terms brought by the second reflection, these additional terms are entirely negligible.

\subsubsection{Taking advantage of the symmetries}

According to the previous results, the truncated model of total currents (20) in presence of the object can be detailed as follows :

$$
\begin{aligned}
I & =I^{(0)}+I^{(1)}+I^{(2)} \\
& =I^{(0)}+I_{a x}^{(1)}+I_{\text {lat }}^{(1)}+I_{a x}^{(2)}+I_{\text {lat }}^{(2)}
\end{aligned}
$$

Then, let us notice that the currents $I^{(0)}+I_{a x}^{(1)}+I_{a x}^{(2)}$ are axisymmetric. To convince oneself of that, we note that $I^{(0)}+I_{a x}^{(2)}$ is generated by differences of uniform potentials applied onto the rings $\mathcal{E}_{\alpha}$ (imposed by the control, for $I^{(0)}$, or by the potential reflected by the object for $I^{(2)}$ ). Similarly, $I_{a x}^{(1)}$ presents the same symmetry but for different reasons. In this case it is the flow of the field $\mathbf{E}_{1}$ reflected by the object and projected in parallel to the axis which creates the current. Also, this field being uniform on the rings and $\mathbf{n}_{\|}$being axisymmetric, $I_{a x}^{(1)}$ is axisymmetric too. Regarding the component $I_{\text {lat }}^{(1)}$, it also obeys a symmetry property. $I_{\text {lat }}^{(1)}$ represents the lateral flow 
of the field $\mathbf{E}_{1}$ reflected by the object and in particular, taking into account the fact that $\mathbf{E}_{1}$ can be reasonably considered as uniform on every ring, we have for any $k=0,1 \ldots n$ :

$$
I_{l a t, k}^{(1)}=\gamma \int_{e_{k}} \mathbf{E}_{1} \cdot \mathbf{n}_{\perp} d s \simeq \gamma \mathbf{E}_{1} \cdot \int_{e_{k}} \mathbf{n}_{\perp} d s .
$$

Thus, if every ring is pairwise divided into opposed identical angular segments, then for every pair of azimuthally opposed $e_{i}$, the measured currents have the same strength but are of opposite sign. Moreover, due to $(55)$ and $S_{\perp}=\operatorname{diag}\left(s_{\perp \alpha(k)}\right), I_{\text {lat }}^{(2)}$ and finally $I_{\text {lat }}=I_{\text {lat }}^{(1)}+I_{\text {lat }}^{(2)}=\left(1+S_{\perp}\right) I_{\text {lat }}^{(1)}$ inherit the same symmetry property. Consequently, the currents of $I_{\text {lat }}$ follow a luminous analogy, the surface of the electrodes facing a conducting object being lit by currents flowing in, while the electrodes on the opposite side of the sensor are in the shadow of the outward currents. Conversely, with an insulating object the nearer side of the sensor is shadowed while the opposite side is in the light. Furthermore, it is possible to extract $I_{\text {lat }}$ from $I$. To do this, we just have to perform the difference of the currents crossing the couples of azimuthally opposed sections $e_{i}$ since, the other components of $I$ being axisymmetric, their difference is null. Finally, since the emission electrode $e_{0}=\mathcal{E}_{0}$ is not divided into opposed sections, such an operation can be encoded in the projection matrix $P_{-}$of dimensions $((n / 2)+1) \times(n+1)$, which once applied to $I=\left(I_{0}, I_{1}, I_{2}, \ldots I_{n}\right)^{T}$, gives the reduced vector : $\left(I_{0}, I_{2}-I_{1}, I_{4}-I_{3}, \ldots I_{n}-I_{n-1}\right)^{T}$, in the case where the numbering of the electrodes $e_{i}(i>1)$, is such that the $\left(e_{i}, e_{i+1}\right)$ are opposed pairs in the same $\mathcal{E}_{\alpha}$. In addition, as the current intensities $\left|I_{i}\right|$ and $\left|I_{i+1}\right|$ are equal it becomes easy to recover the currents vector $I_{\text {lat }}$ by applying a distribution matrix $D_{-}$to the left of $P_{-}$in such a manner that $D_{-} P_{-}=1_{(n+1) \times(n+1)}$. This can be summarized by the extraction formula of the lateral currents from the total currents :

$$
I_{l a t}=D_{-} P_{-} I \text {. }
$$

Moreover, the axisymmetric geometry of the sensor also imposes for every $\mathcal{E}_{\alpha}$ :

$$
\begin{aligned}
\sum_{k(\alpha)} I_{l a t, k(\alpha)}^{(1)} & \simeq \gamma \mathbf{E}_{1} \cdot \sum_{k(\alpha)} \int_{e_{k(\alpha)}} \mathbf{n}_{\perp} d s \\
& =\gamma \mathbf{E}_{1} \cdot \int_{\mathcal{E}_{\alpha}} \mathbf{n}_{\perp} d s=\mathbf{E}_{1} \cdot \mathbf{0}=0,
\end{aligned}
$$

which allows one to write from (55) the matrix relation :

$$
P_{+} I_{l a t}=0 .
$$

Now if we introduce $I_{a x}$, the vector of the axial currents of the sensor, it becomes possible to set it apart by the simple relation :

$$
I_{a x}=I-I_{l a t}=D_{+} P_{+} I .
$$

Finally, taking advantage of the symmetry properties of the sensor allows one to separate the measured currents into two types : the axial components and the lateral components. In the following we will detail further the model of these components.

\subsubsection{Axial model of the currents in the approximation of the second reflection}

If we project (56) onto the electrodes $\mathcal{E}_{\alpha}$, by virtue of (60) the lateral currents disappear and we have at the leading order (with $\left|I_{a x}^{(1)} / I_{a x}^{(2)}\right|=O(R / l)$ ) :

$$
\begin{aligned}
P_{+} I & =\bar{I}^{(0)}+\bar{I}^{(2)}+P_{+} I_{a x}^{(1)} \\
& \simeq \bar{I}^{(0)}+\bar{I}^{(2)}=\left(\bar{C}^{(0)}-\bar{C}^{(0)} K \bar{C}^{(0)}\right) U .
\end{aligned}
$$


Now, if we introduce $\bar{R}^{(0)}$, the axial resistance matrix of the sensor with no object which verifies $1=\bar{C}^{(0)} \bar{R}^{(0)}, 1$ being the $(m+1) \times(m+1)$ identity matrix, we recognize in $(62)$ the first order approximation of the total resistance matrix of the scene ${ }^{5}$, which we denote $\bar{R}$. In fact :

$$
\begin{aligned}
\bar{R}^{-1} & \left.\simeq \bar{R}^{(0)}+K\right)^{-1} \\
& \simeq \bar{C}^{(0)}-\bar{C}^{(0)} K \bar{C}^{(0)} \triangleq \bar{C}^{(0)}+\bar{C}^{(1)}
\end{aligned}
$$

where we denote $\bar{C}^{(1)}$ the conductance perturbation caused by the introduction of a small object into the scene. It follows that in the case of a unique object, the matrix $K$ is nothing but the resistive perturbation (which we denote $\bar{R}^{(1)}$ ) induced by the presence of the object. For instance, if the object is an insulating sphere then $\chi<0$ in (42), and $K$ is then positive, so increasing the total resistance of the scene. Conversely, if the object is a conductive object, $\chi>0$, and its presence decreases the total resistance of the scene. Moreover, by virtue of the superimposition principle, if the objects are far enough from each other to not influence themselves electrically, every object will create a perturbation of the same nature, the whole perturbation being identified as the sum of the individual perturbations. And so we obtain for $p$ objects :

$$
\bar{R}=\bar{R}^{(0)}+\bar{R}^{(1)}=\bar{R}^{(0)}+\sum_{i=1}^{p} K_{i}
$$

where $\bar{R}^{(1)}$ denotes now the perturbative resistance induced by the direct reflection of all the objects existing in the scene. Continuing with the framework of the reflection method, the possible electric influences between the objects can be interpreted on $\bar{R}$ by the addition of coupling resistances which would be approximated by the reflections between the objects. Furthermore, each small object introducing a strong attenuation factor, the effect of the reflections between objects can be neglected except when the objects are in contact. But in this case the electric continuity makes of the objects a unique one so leading one to the case of a unique object.

\subsubsection{Model of the lateral currents in the approximation of the second reflection}

The model of the lateral currents is complementary to the previous one. To measure it, we can apply (58). It then remains to equate those measurements to their expressions obtained from the previous modeling, to obtain :

$$
I_{\text {lat }}=\left(1+S_{\perp}\right) I_{\text {lat }}^{(1)}=\left(1+S_{\perp}\right) L_{l a t} \bar{C}^{(0)} U
$$

with 1 the identity matrix. Finally, when we have several objects in the scene, for similar reasons to those previously evoked, one can neglect their mutual interactions. Also, the (direct) reflections which every object reflects summate and we obtain $L_{l a t}=\sum_{i=1}^{p} L_{l a t, i}$, where every $L_{l a t, i}$ encodes the direct reflection rejected by the $i^{t h}$ object which the sensor receives laterally.

\section{Application to the analytical modeling of the 7-electrode slen- der sensor}

In this section, we apply the previous modeling approach to the case of the 7-electrode slender sensor of Fig. 3 and compare its predictions in ideal unbounded conditions with the BEM simulations.

5. Let us recall that for any invertible matrix $M$ first order perturbated with $\delta M:(M+\delta M)^{-1}=M^{-1}-$ $M^{-1} \delta M M^{-1}+O\left(\|\delta M\|^{2}\right)$ 


\subsection{Experimental conditions}

The sensor of Fig. 3 and 7 is such that $m=3$ and $n=6$ while its dimensions are $: l=22 \mathrm{~cm}$, $l_{\mathcal{E}_{\alpha}}=R=1 \mathrm{~cm}$ for $\alpha=0,1,2,3 ; l_{\mathcal{I}_{1}}=13 \mathrm{~cm}, l_{\mathcal{I}_{2}}=l_{\mathcal{I}_{3}}=2.5 \mathrm{~cm}$. We place in the environment an object of small dimension in comparison with the sensor length (see Fig. 7). The object is a sphere of radius $a=R / 2=0.5 \mathrm{~cm}$ which we move along a parallel or orthogonal line to the sensor axis (see Fig. 7). While the sphere is moved from $-\infty$ to $+\infty$ along this line, the currents entering into the electrodes are measured on each position with the sensor set to a constant voltage $(1 \mathrm{~V}$ for the emitter and $0 \mathrm{~V}$ for the receivers). In order to qualify the accuracy of the analytical model, the experiment is repeated with the BEM simulator. In both cases, the water conductivity is set to $\gamma=0.04 \mathrm{~S} / \mathrm{m}$.

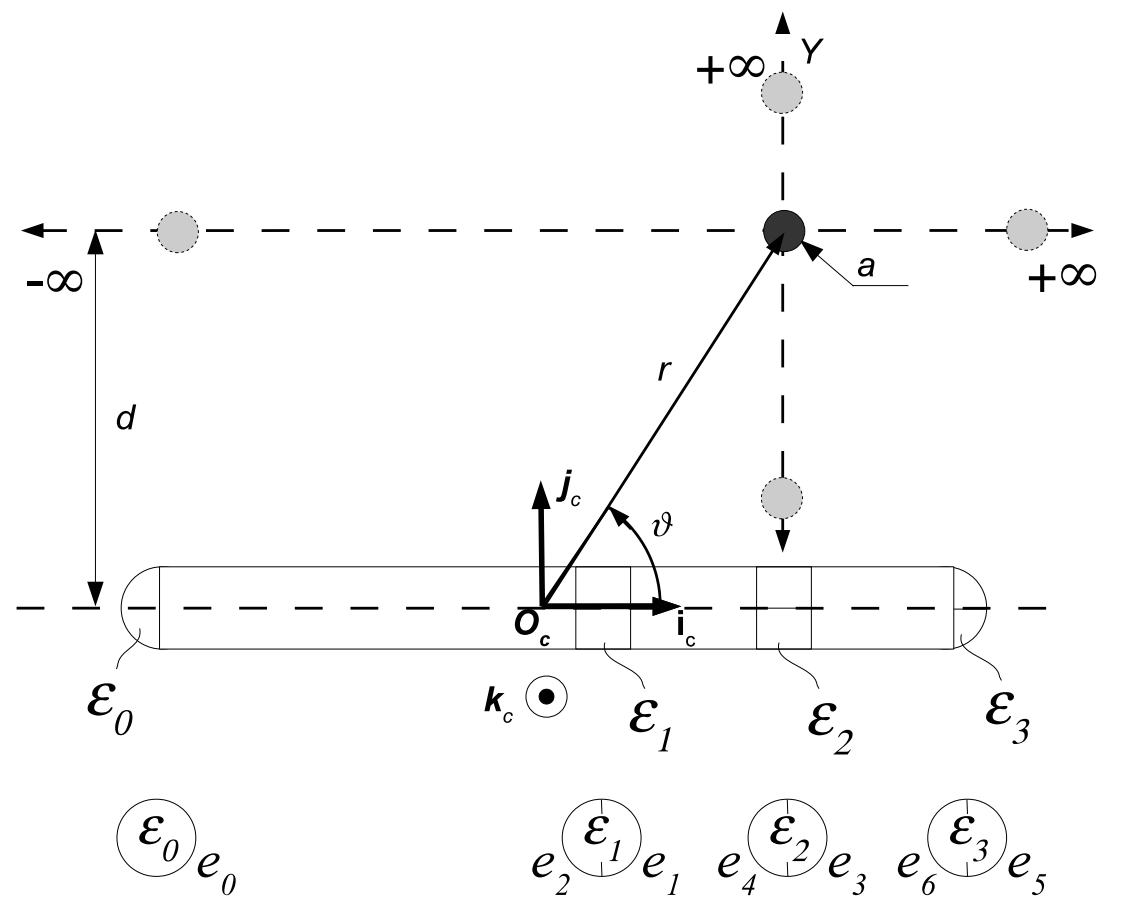

Figure 7 - (top) Schematic view of the benchmark: To test our models we have submitted the sensor to a perturbation by a small sphere. The measurements are compared with both the BEM simulator and the model for different positions of the sphere parallel and perpendicular to the sensor axis. (bottom) The geometry and numbering of measurement electrodes.

\subsection{Results}

Fig. 8 and 9 show the currents measured by the BEM and the model when the sphere is moved from $-\infty$ to $+\infty$ parallel to the sensor axis at distance $d$. In the BEM, the lateral currents are deduced from $I$ invoking $I_{\text {lat }}=D_{-} P_{-} I$, and reported on Fig. 8 for $d=5 \mathrm{~cm}$ and $d=8$ $\mathrm{cm}$ respectively. Due to (55), in both cases the analytical model of (65) and (45) is used with $\left(1+s_{\perp \alpha(k)}\right) \bar{A}_{k}$ replacing $\bar{A}_{k}$ and $1+s_{\perp \alpha(k)}$ a shape factor, deduced from a preliminary calibration (here $d=5 \mathrm{~cm}$ for which we found $1+s_{\perp \alpha(k)}=3.5$ if $e_{k}$ is a half-ring and $1+s_{\perp \alpha(k)}=3.5$, if $e_{k}$ a quarter of sphere). Besides this calibration, the geometry of $I_{l a t}$ is adequately modeled by the reflection axial matrix $L$. In particular, on each of the rings, the maximum (or minimum) of signal appears when the sphere faces the electrode $\left(X=X_{\alpha}\right)$. As regards the axial currents, they are deduced from $I_{a x}=I-I_{l a t}$. Then we calculate $I_{a x}^{(2)}+I_{a x}^{(1)}=I_{a x}-I^{(0)}$ (Fig. 9), where $I^{(0)}$ was first evaluated once and for all with no object in the scene. The results presented in 
Fig. 10(a) and 10(b) show the currents measured for the second test (i.e. when the sphere is moved from $15 \mathrm{~mm}$ to $+\infty$ along a perpendicular line to the sensor axis in front of the electrodes $e_{i=3,4}$ ). The next plots reported on Fig. 10(a) present the lateral currents measured by $e_{i=3,4}$ for different positions of the sphere along this trajectory. Note that $\mathcal{E}_{2}=e_{3} \cup e_{4}$ faces the sphere. Finally, Fig. 10(b) presents the axial current measured by the ring $\mathcal{E}_{2}$ for different positions of the sphere along the lateral trajectory.
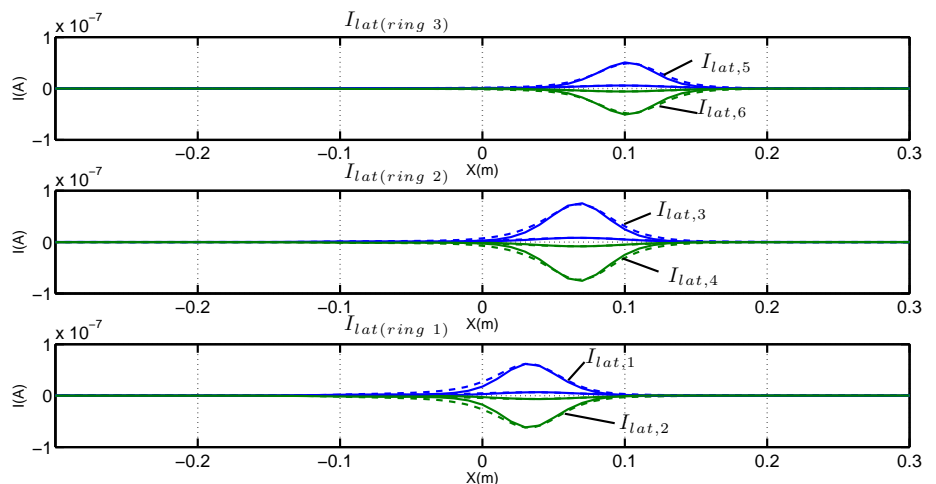

Figure 8 - Currents $I_{\text {lat }}$ in function of $X$ at $Y=d=5$ and $7 \mathrm{~cm}$ measured by the electrodes $e_{i=1,2, . .6}$, (solid lines : model, dotted lines : BEM).
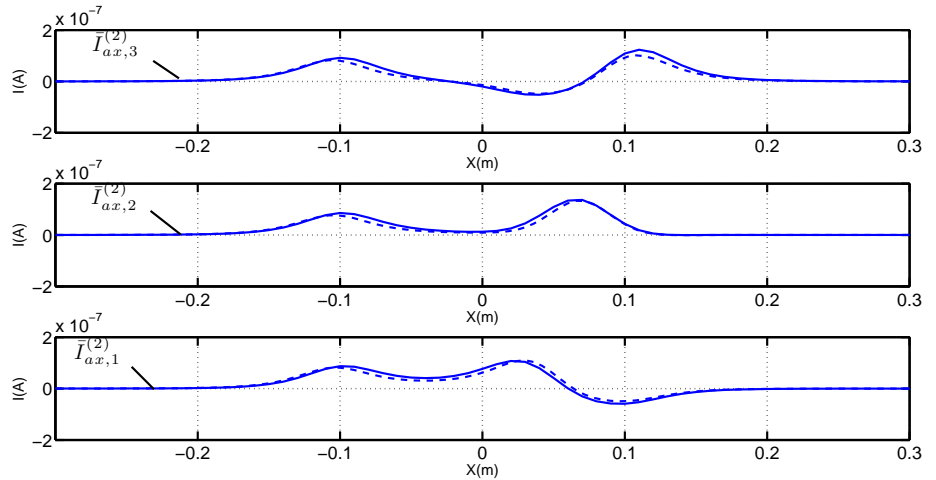

Figure 9 - Currents $I_{a x}$ in function of $X$ at $Y=d=5$ and $7 \mathrm{~cm}$ measured by $\mathcal{E}_{\alpha=1,2,3}$, (solid lines : model, dotted lines : BEM).

\section{Experimental electrolocation of a sphere with the 7-electrode sensor}

In this section we present a preliminary test whose purpose is to illustrate the applicability of the reduced analytical model to real-time electrolocation in robotics.

\subsection{The experimental test bench}

The test is performed on an experimental test bench pictured in Fig.11. This set-up is a cubic $1 \mathrm{~m}^{3}$ tank on which is placed a Cartesian robot that moves in the two directions of the horizontal 


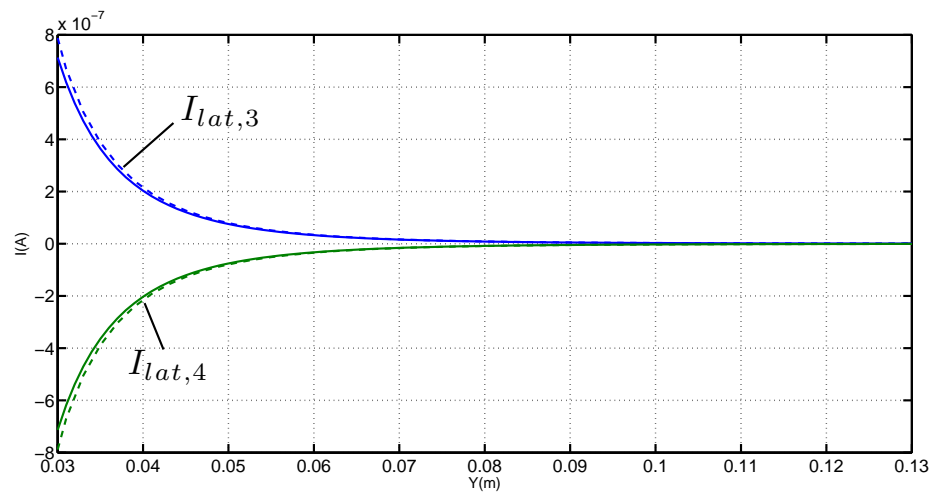

(a) Currents of $I_{\text {lat }}$ in function of $Y$ measured by the electrodes $e_{i=3,4}$.

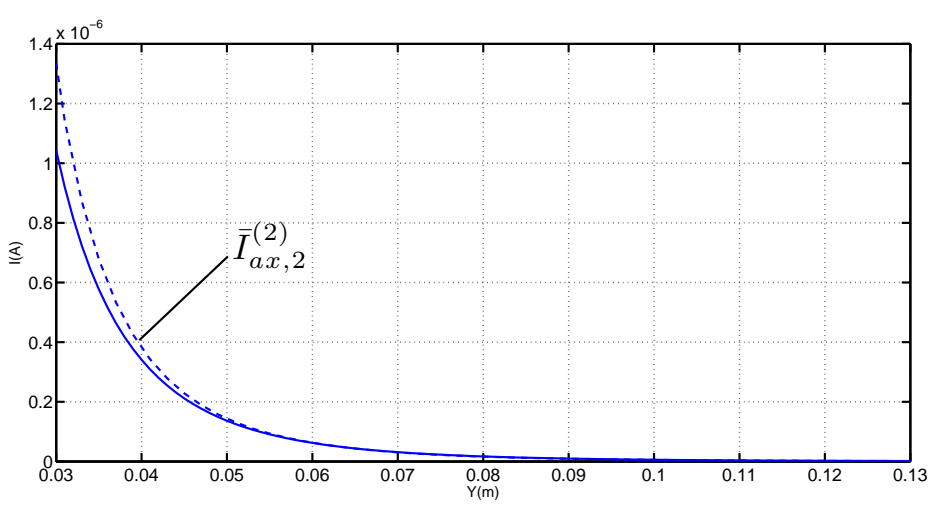

(b) Current of $I_{a x}$ in function of $Y$ measured by the electrode $\mathcal{E}_{2}$

FIgURE 10 - Currents measured by the electrodes $\mathcal{E}_{2}$, (solid lines : model, dotted lines : BEM).

plane and rotates a vertical stick at whose tip the probe is fixed. This device is controlled and monitored with a dSPACE ${ }^{\circledR}$ system which allows the movement of the probe to be controlled in real-time using the exteroceptive feedback of the electric measurements. By placing objects of different shapes and electric properties we can test our electrolocation algorithms in various situations.

\subsection{Conditions and results of the experiment}

The aim of this experimental test is to use the previous analytical model (of the 7- electrode) with a Kalman filter (detailed in simulation in [10]) in order to reconstruct the geometric parameters of a sphere. As in the previous section, a sphere (insulating or conductive) is placed in the plane of the sensor which is constrained to move forward parallel to its axis with a constant axial velocity $V=0.1 \mathrm{~m} / \mathrm{s}$ and no yawing velocity, i.e. $\Omega=0 \mathrm{rad} / \mathrm{s}$. The parameters of the scene are : the radius $a$ of the sphere, the length $r=\|\mathbf{r}\|$ of the vector linking the center of the sphere and that of the probe, and the angle between $\mathbf{r}$ and the sensor axis (see figure 7 ). In order to reconstruct these parameters, the Kalman filter is used with as inputs $V$ and $\Omega$ as well as the axial component $I_{a x}$ of the measured currents while the outputs are the estimated parameters of the scene $\hat{r}, \hat{\vartheta}$ and $\hat{a}$. As regards the initialization of the filter, the variation of $\left|I-I^{(0)}\right|$ up to a given threshold activates the detection of the presence of an object in the sensor's surroundings. Then, since the model of the lateral currents is unable to distinguish between an insulating object and a conductive object situated at the same distance from the sensor axis on its two sides while 


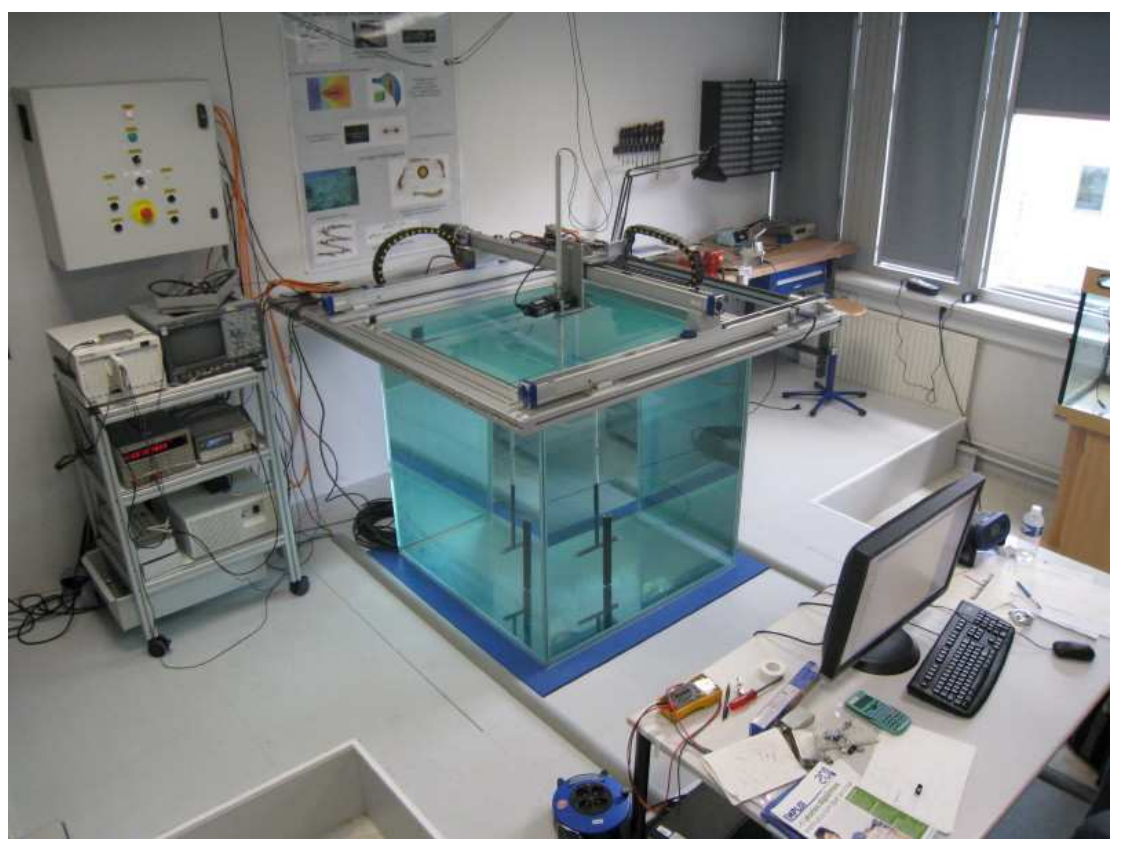

Figure 11 - The experimental set-up. The cartesian robot permits a movement in an horizontal plane with a precision equal to $0.1 \mathrm{~mm}$.

the axial model can do it, $I_{a x}$ is used first to assess the two possible lateral positions and the electric nature of the object. Then, once the electric properties of the object are known, $I_{l a t}$ can be used to determine on which side of the sensor the object is. All of these preliminaries could be used to restrict the search space in a static optimization phase which allows an initial state of the filter to be computed. In the test presented below, the sphere is insulating $(\chi=-1 / 2)$, while the initial estimated and actual states of the scene are fixed to be $: r=0.25 \mathrm{~m}, \vartheta=0.266$ $\operatorname{rad}$ and $a=0.0305 \mathrm{~m}$ and $\hat{r}=0.32 \mathrm{~m}, \hat{\vartheta}=0.336 \mathrm{rad}$ and $\hat{a}=0.002 \mathrm{~m}$. Figure 12 shows the state of the actual and the estimated sphere with respect to time. We can see that the state reconstructed by the filter does converge toward the actual one while figure 13 represents the actual and the estimated scene at different times during the test.
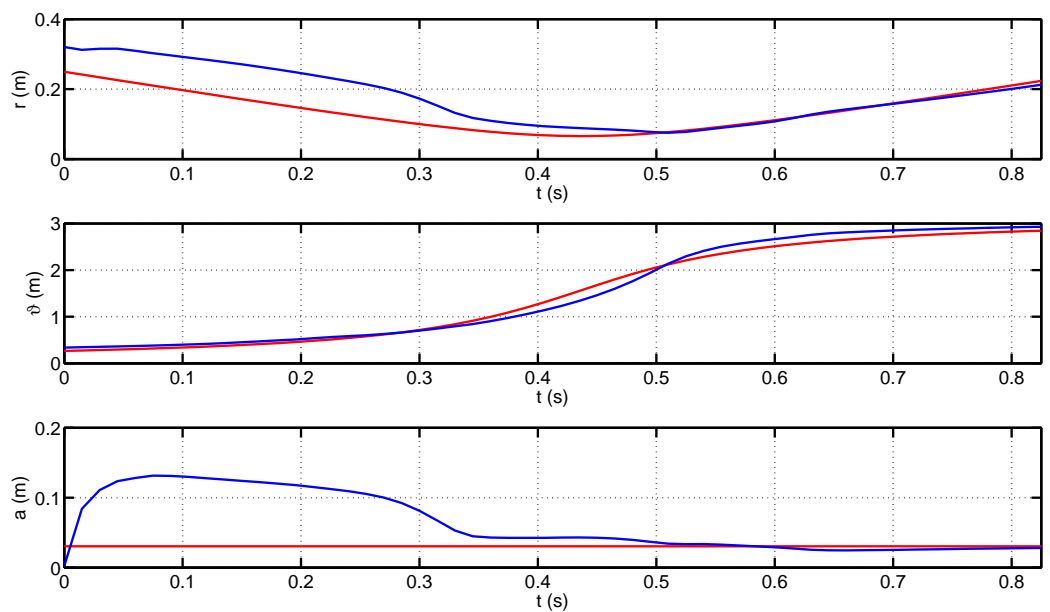

FiguRE 12 - Evolution of the actual (red) and estimated (blue) states of the sphere. 

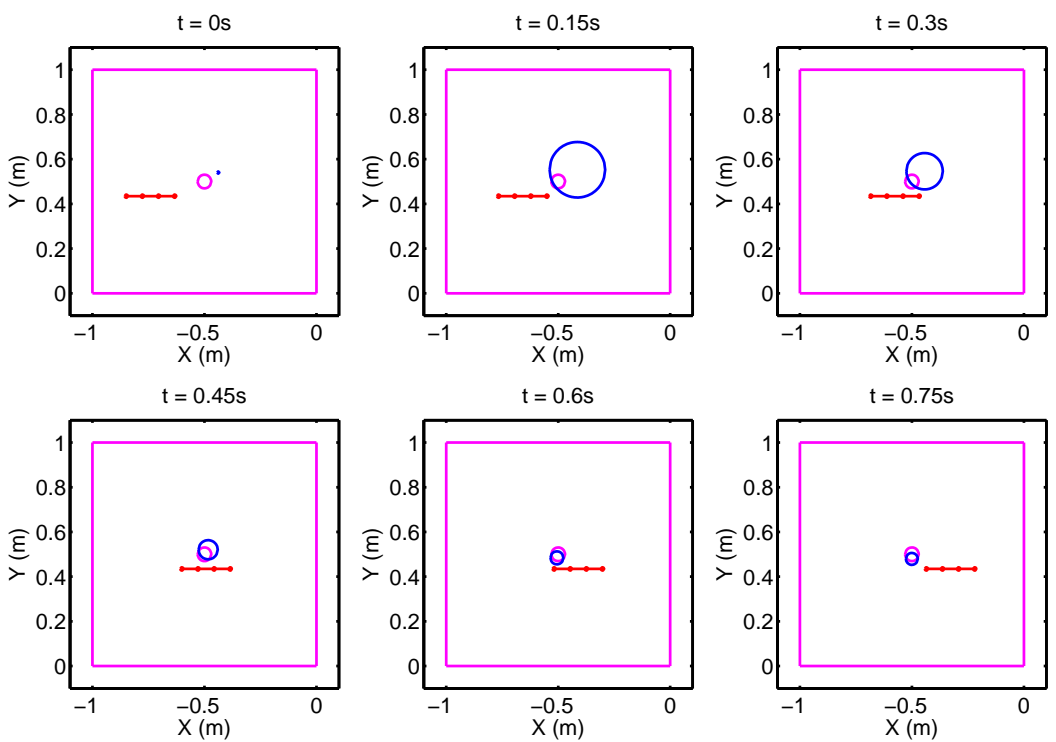

Figure 13 - Actual and estimated scene.

\section{Concluding remarks}
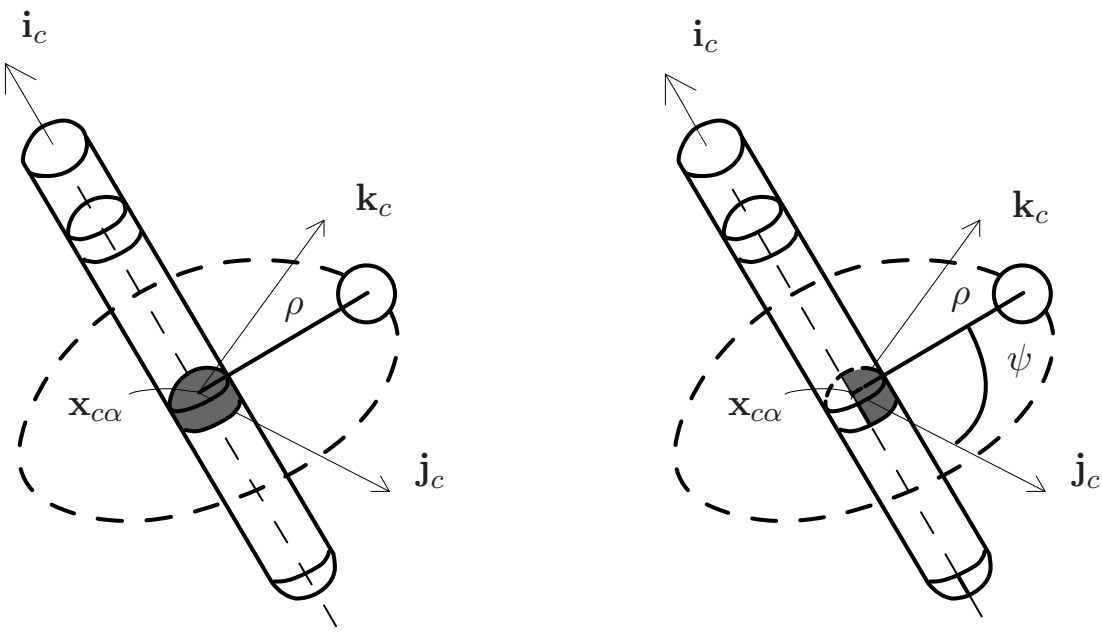

Figure 14 - The role played by the components $I_{a x}$ (left) and $I_{\text {lat }}$ (right) for electrolocation. $I_{a x}$ is the stronger component of the current perturbation, and gives information on the height along direction $\mathbf{i}_{c}$ and the distance $\rho$ from the sensor axis at which we can locate the object while $I_{\text {lat }}$ disambiguates the azimuth angle $\psi$.

As demonstrated in the two previous sections, the analytical reduction scheme is in good agreement with the reference BEM, while being tractable for real-time electrolocation. Beyond these pragmatic aspects, it gives new insights which are useful for the understanding of the electric sense. First, in the context of the reflection method, the presence of an object in the scene brings two contributions to the total measured currents. The first one, which we have denoted $I^{(1)}$, is produced by the electric field reflected by the object $\mathbf{E}_{1}$ which penetrates the geometry of the 
sensor laterally while it is removed from the scene. The second, $I^{(2)}$, is produced by the sensor in order to recover its electric equilibrium while it is submitted to the reflected potential $\phi_{1}$. These two contributions require from the sensor the knowledge of $\mathbf{E}_{0}$, the conductance matrix $\bar{C}^{(0)}$, which encodes the model of the measured currents in the absence of an object, as well as the apparent areas $\left(1+s_{\perp \alpha(k)}\right) \bar{A}_{k}$ of the receiving electrodes $e_{k}$, through which the lateral currents flow, and where the $s_{\perp}$ factors model the lateral polarizability of the electrodes. Moreover, while $\mathbf{E}_{0}$ is approximated analytically, $\bar{C}^{(0)}$ and $s_{\perp \alpha(k)}$ can be measured once for all in a preliminary calibration phase. The other parameters of the model depend only on the geometry of the scene and obey simple relations which are easily usable for online calculations, such as those required by observer based electrolocation algorithms. Exploiting the symmetries of the sensor, $I_{a x}$ and $I_{\text {lat }}$ can be easily extracted from the total currents vector $I$ by simple arithmetic combinations of its components. In further detail, $I_{a x}$ encodes the response in currents of the sensor to the axial voltage imbalance due to the induced potential $\phi_{1}$, while $I_{\text {lat }}$ encodes the lateral polarization of the rings induced by the presence of the object. That being said, it becomes obvious that if $(\rho, \psi, X)$ represent the cylindrical coordinates of the object center in the frame attached to the sensor, then as $I_{a x}$ does not depend on the azimuth angle $\psi$, the same object placed anywhere on a circle perpendicular to the sensor axis and centered on it, will produce the same $I_{a x}$ whereas $I_{l a t}$ is richer since it depends on all three coordinates (see Fig. 14). Pursuing this discussion further, the lateral currents obey purely geometric projection laws as suggested by the biologists [16],[17]. In particular, the object is simply located on the axis perpendicular to the surface of the sensor and centered in the area of maximum intensity of the transcutaneous measures. This advantage will be exploited in future to address the problem of electrolocation. We can also mention that the second contribution $I_{a x}$ is nothing but the so-called "polyspherical model" of the currents obtained in [8] or [9] with a simplified sensor geometry where all the electrodes are considered as simple spheres and where the role of the insulators is modeled through the effective radius of the spheres. Finally, the combination of the axial and lateral models offers numerous strategies for electrolocation that we are now exploring. All together, we believe the reduction scheme presented in the article constitutes a real breakthrough towards rigorous and quick modeling of electrolocation problems in robotics.

\section{Appendix A : Proof of (38)}

To continue the reduction of (37), we will use the following notations : $\mathbf{x}$ will denote a point on the border of the sensor of abscissa $X$ along the symmetry axis of the sensor (positively measured from the extremity of the hemisphere $\left.\mathcal{E}_{0}\right)$ and $\mathbf{x}_{\perp}(X)$ will represent its orthogonal projection to the axis of the sensor. A lateral section centered on $\mathbf{x}_{\perp}(X)$ will be denoted $\mathcal{C}_{X}$ while the center of $\mathcal{E}_{\alpha}$ will be denoted $\mathbf{x}_{c \alpha}, X_{\alpha}$ being the abscissa of the central section of $\mathbf{x}_{c \alpha}$ on the sensor axis (see Fig. 3). Then, exploiting the axisymmetry of the emitting electrodes and the insulating surfaces it becomes natural to write in (37) :

$$
\begin{aligned}
\int_{\mathcal{E}_{\alpha}} \cdot d s & =\int_{\mathcal{L}_{\mathcal{E} \alpha}}\left(\int_{\mathcal{C}_{X}} \cdot d \mathcal{C}_{X}\right) d X, \\
\int_{\mathcal{I}_{\alpha}} \cdot d s & =\int_{\mathcal{L}_{\mathcal{I}_{\alpha}}}\left(\int_{\mathcal{C}_{X}} \cdot d \mathcal{C}_{X}\right) d X,
\end{aligned}
$$

where $\mathcal{L}_{\mathcal{E} \alpha}=\left[X_{\alpha}-\left(l_{\mathcal{E} \alpha} / 2\right), X_{\alpha}+\left(l_{\mathcal{E} \alpha} / 2\right)\right], \mathcal{L}_{\mathcal{I}_{\alpha}}=\left[X_{\alpha}-\left(l_{\mathcal{I}_{\alpha}} / 2\right), X_{\alpha}+\left(l_{\mathcal{I}_{\alpha}} / 2\right)\right]$ while $d \mathcal{C}_{X}=$ $R d \psi$, and $\psi$ denotes the azimuth angle of the sensor cylindrical coordinates : $\left(x_{1}, x_{2}, x_{3}\right)=$ $(X, \rho c \psi, \rho s \psi)$. Now, let us apply a multipolar expansion to the three integrals of (37) that we 
rewrite $4 \pi \phi_{0}(\mathbf{y})=\mathcal{J}_{1}-\mathcal{J}_{2}-\mathcal{J}_{3}$. We obtain for the first of them :

$$
\begin{array}{r}
\mathcal{J}_{1}=\sum_{\alpha=0}^{m} \int_{\mathcal{L}_{\mathcal{E} \alpha}}\left(\int_{\mathcal{C}_{X}} \frac{\partial \phi_{0}}{\partial n}\left(\frac{1}{r}+\frac{x_{i}^{\prime} x_{i}}{r^{3}}\right) d \mathcal{C}_{X}\right) d X+ \\
\sum_{\alpha=0}^{m} \int_{\mathcal{L}_{\mathcal{E} \alpha}}\left(\int_{\mathcal{C}_{X}} \frac{\partial \phi_{0}}{\partial n}\left(\frac{1}{2}\left(-\frac{\delta_{i j}}{r^{3}}+3 \frac{x_{i} x_{j}}{r^{5}}\right) x_{i}^{\prime} x_{j}^{\prime}\right) d \mathcal{C}_{X}\right) d X,
\end{array}
$$

where $\left(x_{1}, x_{2}, x_{3}\right)$ and $\left(x_{1}^{\prime}, x_{2}^{\prime}, x_{3}^{\prime}\right)$ denote respectively the coordinates of $\mathbf{y}-\mathbf{x}_{\perp}(X)=\mathbf{r}$ and $\mathbf{x}-\mathbf{x}_{\perp}(X)$ in the frame attached to the section $\mathcal{C}_{X}$ of center $\mathbf{x}_{\perp}(X)$. By integrating on the cross sections, one obtains :

$$
\begin{aligned}
\mathcal{J}_{1} & =\sum_{\alpha=0}^{m} \int_{\mathcal{L}_{\mathcal{E}_{\alpha}}}\left(\frac{1}{r} l^{(0)}+l_{i}^{(1)}\left(\frac{x_{i}}{r^{3}}\right)\right) d X \\
& +\sum_{\alpha=0}^{m} \int_{\mathcal{L}_{\mathcal{E}_{\alpha}}}\left(\frac{1}{2}\left(-\frac{\delta_{i j}}{r^{3}}+3 \frac{x_{i} x_{j}}{r^{5}}\right) l_{i j}^{(2)}\right) d X,
\end{aligned}
$$

the expression in which we introduced the cross-sectional moments of the distribution of currents :

$$
\begin{aligned}
l^{(0)} & =\int_{\mathcal{C}_{X}} \frac{\partial \phi_{0}}{\partial n} d \mathcal{C}_{X}, l_{i}^{(1)}=\int_{\mathcal{C}_{X}} \frac{\partial \phi_{0}}{\partial n} x_{i}^{\prime} d \mathcal{C}_{X}, \\
l_{i j}^{(2)} & =\int_{\mathcal{C}_{X}} \frac{\partial \phi_{0}}{\partial n} x_{i}^{\prime} x_{j}^{\prime} d \mathcal{C}_{X},
\end{aligned}
$$

where $\gamma l^{(0)}$ represents the currents density crossing the electrodes per unit of sensor length in the absence of an object. In the following, this current density is denoted $j_{\alpha}^{(0)}$ when it refers to the ring $\mathcal{E}_{\alpha}$ (i.e. $\mathcal{C}_{X} \subset \mathcal{E}_{\alpha}$ ) and verifies :

$$
\int_{\mathcal{L}_{\mathcal{E}_{\alpha}}} j_{\alpha}^{(0)} d X=\bar{I}_{\alpha}^{(0)}
$$

Moreover, due to the axisymmetry of the sensor boundary and of its polarization, $\phi_{0}$ and then $\partial \phi_{0} / \partial n$ are functions of the axial variable $X$ only. Thus, we can extract them from the integrals (68) and rewrite (67) as :

$$
\begin{aligned}
\mathcal{J}_{1} & =\sum_{\alpha=0}^{m} \int_{\mathcal{L}_{\mathcal{E}_{\alpha}}}\left(\frac{\partial \phi_{0}}{\partial n}\right)\left(\frac{1}{r} m^{(0)}+m_{i}^{(1)}\left(\frac{x_{i}}{r^{3}}\right)\right) d X \\
& +\sum_{\alpha=0}^{m} \int_{\mathcal{L}_{\mathcal{E}_{\alpha}}}\left(\frac{1}{2}\left(-\frac{\delta_{i j}}{r^{3}}+3 \frac{x_{i} x_{j}}{r^{5}}\right) m_{i j}^{(2)}\right) d X,
\end{aligned}
$$

where we have introduced the multipolar moments of the sections geometry :

$$
\begin{aligned}
& m^{(0)}=\int_{\mathcal{C}_{X}} d \mathcal{C}_{X}=2 \pi R, \\
& m_{i}^{(1)}(X)=\int_{\mathcal{C}_{X}} x_{i}^{\prime} d \mathcal{C}_{X}=O\left(R^{2}\right), \\
& m_{i j}^{(2)}(X)=\int_{\mathcal{C}_{X}} x_{i}^{\prime} x_{j}^{\prime} d \mathcal{C}_{X}=O\left(R^{3}\right) .
\end{aligned}
$$

Then, because the first order moments are null and those of the following order ones are proportional to $O\left(R^{3}\right)$ we have :

$$
\mathcal{J}_{1}=\sum_{\alpha=0}^{m} \int_{\mathcal{L}_{\mathcal{E}_{\alpha}}} \gamma^{-1} j_{\alpha}^{(0)}\left(\frac{1}{r}+O\left(\frac{R^{3}}{r^{3}}\right)\right) d X
$$


If we re-apply a multipolar expansion to the leading order term of (72) but this time with respect to the axial dimension $X$, we obtain by writing $r_{\alpha}=\left\|\mathbf{y}-\mathbf{x}_{c \alpha}\right\|$ and by denoting $x_{1}$ the projection of $\mathbf{y}-\mathbf{x}_{c \alpha}$ on the axis of the sensor :

$$
\begin{aligned}
& \sum_{\alpha=0}^{m} \int_{\mathcal{L}_{\mathcal{E}_{\alpha}}} \gamma^{-1} j_{\alpha}^{(0)} \frac{1}{r(X)} d X= \\
& \sum_{\alpha=0}^{m} \int_{\mathcal{L}_{\mathcal{E}_{\alpha}}} \gamma^{-1} j_{\alpha}^{(0)}\left(\frac{1}{r_{\alpha}}+\frac{x_{1}}{r_{\alpha}^{3}}\left(X-X_{\alpha}\right)\right) d X,
\end{aligned}
$$

and from (69):

$$
\mathcal{J}_{1}=\sum_{\alpha=0}^{m} \frac{1}{\gamma r_{\alpha}} \bar{I}_{\alpha}^{(0)}\left(1+O\left(\frac{l_{\mathcal{E}_{\alpha}}^{2}}{r_{\alpha}^{2}}\right)\right) .
$$

Using once again the fact that the emitting electrodes and the insulating surfaces are axisymmetric, it becomes natural to rewrite the two other terms of (37) as :

$$
\begin{aligned}
& \mathcal{J}_{2}=\sum_{\alpha=0}^{m} U_{\alpha} \int_{\mathcal{L}_{\mathcal{E}_{\alpha}}}\left(\int_{\mathcal{C}_{X}} \frac{\partial(1 / r)}{\partial n} d \mathcal{C}_{X}\right) d X, \\
& \mathcal{J}_{3}=\sum_{\alpha=1}^{m} \int_{\mathcal{L}_{\mathcal{I}_{\alpha}}}\left(\int_{\mathcal{C}_{X}} \phi_{0} \frac{\partial(1 / r)}{\partial n} d \mathcal{C}_{X}\right) d X .
\end{aligned}
$$

When we expand the previous integrals into multipolar moments, we find :

$$
\begin{aligned}
& \mathcal{J}_{2}=\sum_{\alpha=0}^{m} U_{\alpha} \int_{\mathcal{L}_{\mathcal{E}_{\alpha}}}\left(\int_{\mathcal{C}_{X}} \frac{\partial}{\partial n}\left(\frac{1}{r}\right) d \mathcal{C}_{X}\right) d X+ \\
& \sum_{\alpha=0}^{m} U_{\alpha} \int_{\mathcal{L}_{\mathcal{E}_{\alpha}}}\left(\int_{\mathcal{C}_{X}} \frac{\partial}{\partial n}\left(\frac{x_{i}^{\prime} x_{i}}{r^{3}}\right) d \mathcal{C}_{X}\right) d X+ \\
& \sum_{\alpha=0}^{m} U_{\alpha} \int_{\mathcal{L}_{\mathcal{E}_{\alpha}}}\left(\int_{\mathcal{C}_{X}} \frac{\partial}{\partial n}\left(\left(-\frac{\delta_{i j}}{r^{3}}+3 \frac{x_{i} x_{j}}{r^{5}}\right) \frac{x_{i}^{\prime} x_{j}^{\prime}}{2}\right) d \mathcal{C}_{X}\right) d X, \\
& \mathcal{J}_{3}=\sum_{\alpha=1}^{m} \int_{\mathcal{L}_{\mathcal{I}_{\alpha}}}\left(\int_{\mathcal{C}_{X}} \phi_{0} \frac{\partial}{\partial n}\left(\frac{1}{r}+\frac{x_{i}^{\prime} x_{i}}{r^{3}}\right) d \mathcal{C}_{X}\right) d X+ \\
& \sum_{\alpha=1}^{m} \int_{\mathcal{L}_{\mathcal{I}_{\alpha}}}\left(\int_{\mathcal{C}_{X}} \phi_{0} \frac{\partial}{\partial n}\left(\left(-\frac{\delta_{i j}}{r^{3}}+3 \frac{x_{i} x_{j}}{r^{5}}\right) \frac{x_{i}^{\prime} x_{j}^{\prime}}{2}\right) d \mathcal{C}_{X}\right) d X,
\end{aligned}
$$

where $\left(x_{1}, x_{2}, x_{3}\right)$ and $\left(x_{1}^{\prime}, x_{2}^{\prime}, x_{3}^{\prime}\right)$ respectively denote the coordinates of $\mathbf{y}-\mathbf{x}_{\perp}(X)=\mathbf{r}$ and of $\mathbf{x}-\mathbf{x}_{\perp}(X)=\mathbf{r}^{\prime}$ in the frame attached to the section $\mathcal{C}_{X}$ of center $\mathbf{x}_{\perp}(X)$. Again, introducing the multipolar moments in the cross-sections, we obtain :

$$
\begin{aligned}
\mathcal{J}_{2}= & \sum_{\alpha=0}^{m} U_{\alpha} \int_{\mathcal{L}_{\mathcal{E}_{\alpha}}}\left(n_{i}^{(1)}\left(\frac{x^{i}}{r^{3}}\right)\right) d X+ \\
& \sum_{\alpha=0}^{m} U_{\alpha} \int_{\mathcal{L}_{\mathcal{E}_{\alpha}}}\left(\frac{1}{2} n_{i j}^{(2)}\left(-\frac{\delta_{i j}}{r^{3}}+3 \frac{x_{i} x_{j}}{r^{5}}\right)\right) d X,
\end{aligned}
$$

where we have introduced the normal multipolar moments of the cross-sections :

$$
\begin{aligned}
n_{i}^{(1)}(X) & =\int_{\mathcal{C}_{X}} \frac{\partial x_{i}^{\prime}}{\partial n} d \mathcal{C}_{X}=O(R), \\
n_{i j}^{(2)}(X) & =\int_{\mathcal{C}_{X}} \frac{\partial\left(x_{i}^{\prime} x_{j}^{\prime}\right)}{\partial n} d \mathcal{C}_{X}=O\left(R^{2}\right) .
\end{aligned}
$$

In the same manner :

$$
\begin{aligned}
\mathcal{J}_{3}= & \sum_{\alpha=1}^{m} \int_{\mathcal{L}_{\mathcal{I}_{\alpha}}} \phi_{0}\left(n_{i}^{(1)}\left(\frac{x^{i}}{r^{3}}\right)\right) d X+ \\
& \sum_{\alpha=1}^{m} \int_{\mathcal{L}_{\mathcal{I}_{\alpha}}} \phi_{0}\left(\frac{1}{2} n_{i j}^{(2)}\left(-\frac{\delta_{i j}}{r^{3}}+3 \frac{x_{i} x_{j}}{r^{5}}\right)\right) d X .
\end{aligned}
$$


Finally, the symmetry of the sections allows to remove the first order geometric moments from (78). Thus, by denoting $l_{\mathcal{I}_{\alpha}}$ the length of every insulating connection cylinder $\mathcal{I}_{\alpha}$, we have :

$$
\begin{aligned}
\mathcal{J}_{2} & =\sum_{\alpha=0}^{m} U_{\alpha} \int_{\mathcal{L}_{\mathcal{E}_{\alpha}}}\left(\frac{1}{2} n_{i j}^{(2)}\left(-\frac{\delta_{i j}}{r^{3}}+3 \frac{x_{i} x_{j}}{r^{5}}\right)\right) d X \\
& =\sum_{\alpha=0}^{m} O\left(U_{\alpha} \frac{R^{2} l_{\mathcal{E}_{\alpha}}}{r_{\alpha}^{3}}\right),
\end{aligned}
$$

But then, with $r_{\alpha}=\left\|\mathbf{y}-\mathbf{x}_{c \alpha}\right\|, U_{\bar{\alpha}}=\sup \left(\left|U_{\alpha-1}\right|,\left|U_{\alpha}\right|\right), U_{\underline{\alpha}}=\inf \left(\left|U_{\alpha-1}\right|,\left|U_{\alpha}\right|\right)$, and since $\forall \mathbf{x} \in \mathcal{I}_{\alpha}: U_{\underline{\alpha}} \leq\left|\phi_{0}(\mathbf{x})\right| \leq U_{\bar{\alpha}}$, we have also :

$$
\begin{aligned}
\mathcal{J}_{3} & =\sum_{\alpha=1}^{m} \int_{\mathcal{L}_{\mathcal{I}_{\alpha}}} \phi_{0}\left(\frac{1}{2} n_{i j}^{(2)}\left(-\frac{\delta_{i j}}{r^{3}}+3 \frac{x_{i} x_{j}}{r^{5}}\right)\right) d X \\
& =\sum_{\alpha=1}^{m} O\left(U_{\bar{\alpha}} \frac{R^{2} l_{\mathcal{I}_{\alpha}}}{r_{\alpha}^{3}}\right) .
\end{aligned}
$$

If by extension $l_{\mathcal{I}_{0}}=0$, then $(37)$ can be rewritten as :

$$
\begin{aligned}
4 \pi \phi_{0}(\mathbf{y})= & \sum_{\alpha=0}^{m}\left(\gamma^{-1} \bar{I}_{\alpha}^{(0)}\left(1+O\left(\frac{l_{\mathcal{E}_{\alpha}}^{2}}{r_{\alpha}^{2}}\right)\right)\right)+ \\
& \sum_{\alpha=0}^{m}\left(O\left(U_{\alpha} \frac{R^{2} l_{\mathcal{E}_{\alpha}}}{r_{\alpha}^{3}}\right)+O\left(U_{\bar{\alpha}} \frac{R^{2} l_{\mathcal{I}_{\alpha}}}{r_{\alpha}^{3}}\right)\right) .
\end{aligned}
$$

Finally, the relative order of magnitude of the $U_{\alpha}$ (or the $U_{\bar{\alpha}}$ ) with respect to the $\bar{I}_{\alpha}^{(0)}$ must be evaluated. It is sufficient to evaluate the order of magnitude of the resistance in absence of object $\bar{R}^{(0)}$ for this. In fact it is possible to show that this order of magnitude is that of a simplified sensor where the ring-shaped electrodes are considered as spheres of radius $R$. But in this case the maximal resistance between the electrodes is the resistance between one electrode and itself for which the Gauss theorem permits one to write $: 4 \pi R^{2} E=q_{\alpha} / \epsilon=\bar{I}_{\alpha}^{(0)} / \gamma$ (with $q_{\alpha}$ the total charge of the electrode $\mathcal{E}_{\alpha}$ and $E$ the intensity of the (normal) field on its surface). Thus, for any couple of electrodes $\left(\mathcal{E}_{\alpha}, \mathcal{E}_{\beta}\right)$, we have : $\bar{R}_{\alpha \beta}^{(0)}<1 /(\gamma R)$, which once used in (81) with $U_{\alpha}=\sum_{\beta=0}^{\beta=m} \bar{R}_{\alpha \beta}^{(0)} \bar{I}_{\beta}^{(0)}$, allows one to write :

$$
\begin{aligned}
4 \pi \phi_{0}(\mathbf{y}) & =\sum_{\alpha=0}^{m} \frac{\bar{I}_{\alpha}^{(0)}}{\gamma r_{\alpha}}\left(1+O\left(\frac{l_{\mathcal{E}_{\alpha}}^{2}}{r_{\alpha}^{2}}\right)+\sum_{\beta=0}^{m} O\left(\frac{R l_{\mathcal{E}_{\beta}}}{r_{\beta}^{2}}\right)\right) \\
& +\sum_{\alpha=0}^{m} \frac{\bar{I}_{\alpha}^{(0)}}{\gamma r_{\alpha}}\left(\sum_{\beta=0}^{m} O\left(\frac{R l_{\mathcal{I}_{\beta}}}{r_{\beta}^{2}}\right)\right) .
\end{aligned}
$$

Since $\sum_{\alpha=0}^{m} l_{\mathcal{E}_{\alpha}}+\sum_{\beta=1}^{m} l_{\mathcal{I}_{\beta}}=l=O\left(r_{\alpha}\right)=O\left(r_{\beta}\right)=O(r)$ we finally obtain (38).

\section{Appendix B : Proof of (44)}

From (43), we notice that an expansion of $\mathbf{E}_{1}$ on each $\mathcal{E}_{\alpha}$ with respect to the dimensions of $\mathcal{E}_{\alpha}$ allows one to write :

$$
I_{k}^{(1)}=-\gamma\left(\nabla \phi_{1}\left(\mathbf{x}_{c \alpha}\right)\right) \cdot \int_{e_{k}} \mathbf{n} d s+O\left(\frac{R}{r}\right)
$$


where $\nabla \phi_{1}\left(\mathbf{x}_{c \alpha}\right)$ is the leading order term of the expansion of $-\mathbf{E}_{1}$ in any point of $e_{k} \subset \mathcal{E}_{\alpha}$. In the following, we will denote $\alpha(k)$ as the index of the ring to which $e_{k}$ belongs. Also, by writing $\int_{e_{k}} \mathbf{n} d s=\bar{A}_{k} \overline{\mathbf{n}}_{k}$ with $\overline{\mathbf{n}}_{k}$ a unit vector which we place at the center of the receiver $e_{k}$ :

$$
I_{k}^{(1)}=-\gamma \bar{A}_{k}\left(\nabla \phi_{1}\left(\mathbf{x}_{c \alpha(k)}\right)\right) \cdot \overline{\mathbf{n}}_{k}+O\left(\frac{R}{r}\right) .
$$

By placing the object frame $\left(\mathbf{y}_{c}, \mathbf{e}_{1}, \mathbf{e}_{2}, \mathbf{e}_{3}\right)$ in order that $\mathbf{p}=p \mathbf{e}_{3}$ (where $p>0$ ) and introducing $r, \theta$ and $\phi$ the spherical coordinates of a point of $\mathcal{E}_{\alpha}$ in such a frame, we obtain from (40): $\phi_{1}=p c \theta f(r)$ with $\theta$ the meridian angle of $\mathbf{x} \in e_{k}$ in the object frame and $f(r)=1 / r^{2}$. Then, because for any function $h$ the normal derivative can be written as following :

$$
\frac{\partial h}{\partial n}=\nabla h \cdot \mathbf{n}=\left(\frac{\partial h}{\partial r} \mathbf{e}_{r}+\frac{1}{r} \frac{\partial h}{\partial \theta} \mathbf{e}_{\theta}+\frac{1}{r s \theta} \frac{\partial h}{\partial \phi} \mathbf{e}_{\phi}\right) \cdot \mathbf{n},
$$

we have :

$$
\begin{aligned}
& I_{k}^{(1)}=-\bar{A}_{k} \gamma \nabla \phi_{1}\left(\mathbf{x}_{c \alpha(k)}\right) \cdot \overline{\mathbf{n}}_{k} \\
= & \gamma \bar{A}_{k} p\left(c \theta_{\alpha(k)} \frac{\partial f}{\partial r} \mathbf{e}_{r \alpha(k)}-s \theta_{\alpha(k)} \frac{f\left(r_{\alpha(k)}\right)}{r_{\alpha(k)}} \mathbf{e}_{\theta \alpha(k)}\right) \cdot \overline{\mathbf{n}}_{k} .
\end{aligned}
$$

Thus, having also $c \theta_{\alpha(k)}=\mathbf{e}_{3} \cdot \mathbf{e}_{r \alpha(k)}$ and $s \theta_{\alpha(k)}=\mathbf{e}_{3} \cdot \mathbf{e}_{\theta \alpha(k)}$ we can write :

$$
\begin{aligned}
I_{k}^{(1)} & \simeq-\gamma \bar{A}_{k} \frac{\partial f}{\partial r}\left(\mathbf{e}_{r \alpha(k)} \cdot \overline{\mathbf{n}}_{k}\right)\left(\mathbf{e}_{r \alpha(k)} \cdot \mathbf{p}\right) \\
& +\gamma \bar{A}_{k} \frac{f\left(r_{\alpha(k)}\right)}{r_{\alpha(k)}}\left(\mathbf{e}_{\theta \alpha(k)} \cdot \overline{\mathbf{n}}_{k}\right)\left(\mathbf{e}_{\theta \alpha(k)} \cdot \mathbf{p}\right) .
\end{aligned}
$$

Then by introducing the notations $\cos _{k}=\overline{\mathbf{n}}_{k} \cdot \mathbf{e}_{r \alpha(k)}$ and $\cos \mu_{k}=\overline{\mathbf{n}}_{k} \cdot \mathbf{e}_{\theta \alpha(k)}$ and $\mathbf{p}=\mathbf{P} . \mathbf{E}_{0}$ we have :

$$
I_{k}^{(1)} \simeq \frac{\bar{A}_{k}}{4 \pi}\left(c \nu_{k} \frac{\partial f}{\partial r} \mathbf{e}_{r \alpha(k)}+c \mu_{k} \frac{f\left(r_{\alpha(k)}\right)}{r_{\alpha(k)}} \mathbf{e}_{\theta \alpha(k)}\right) \cdot \mathbf{P} \cdot \mathbf{E}_{0} .
$$

And as $f(r)=1 / r^{2}$ we obtain, from (39) and with all position vectors pointing from the sensor to the object :

$$
I_{k}^{(1)} \simeq \sum_{\beta=0}^{m} \frac{-\bar{A}_{k}}{4 \pi r_{\alpha(k)}^{3}}\left(2 c \nu_{k} \mathbf{e}_{r \alpha(k)}+c \mu_{k} \mathbf{e}_{\theta \alpha(k)}\right) . \mathbf{P} \cdot\left(\frac{\mathbf{r}_{\beta}}{r_{\beta}^{3}}\right) \bar{I}_{\beta}^{(0)},
$$

which can be rewritten in the matrix form (44).

\section{Appendix C : Proof of (50)}

To prove expression (50), let us first observe that, because of the slenderness of the sensor, the gradient of $\phi_{1}$ which we denote $\mathbf{E}_{1}$, can be expanded in perturbations with respect to the lateral dimensions of each section $\mathcal{C}_{X}$. Also, we can rewrite the first term of the right hand side of (28), where let us remind that $r=\|\mathbf{r}\|=\left\|\mathbf{x}^{\prime}-\mathbf{x}\right\|$ and $\mathbf{x} \in \mathcal{E}_{\alpha}$, as :

$$
\begin{aligned}
& \sum_{\beta=1}^{m} \int_{\mathcal{I}_{\beta}}\left(\frac{\partial \phi_{1}}{\partial n}\right) \frac{1}{r} d s= \\
& \sum_{\beta=1}^{m} \int_{\mathcal{L}_{\mathcal{I}_{\beta}}} \mathbf{E}_{1}\left(\mathbf{x}_{\perp}\left(X^{\prime}\right)\right) \cdot \int_{\mathcal{C}_{X^{\prime}}} \frac{\mathbf{n}}{r} d \mathcal{C}_{X} d X^{\prime}(1+O(R / r)),
\end{aligned}
$$


where $X$ and $X^{\prime}$ denote the abscissa of $\mathbf{x}$ and $\mathbf{x}^{\prime}$ along $\left(O, \mathbf{i}_{c}\right)$ while $l_{X}\left(X^{\prime}\right)=X^{\prime}-X$ and $\xi_{X}\left(X^{\prime}\right)=\left|R / l_{X}\left(X^{\prime}\right)\right|$. Now, by writing : $\mathbf{r}=\mathbf{x}^{\prime}-\mathbf{x}=\left(\mathbf{x}^{\prime}-\mathbf{x}_{\perp}^{\prime}\right)+\left(\mathbf{x}_{\perp}^{\prime}-\mathbf{x}_{\perp}\right)+\left(\mathbf{x}_{\perp}-\mathbf{x}\right)=$ $\mathbf{d}+l_{X}\left(X^{\prime}\right) \mathbf{i}_{c}-\mathbf{d}$, and by taking the cylindrical coordinates of $\mathbf{x}^{\prime}:\left(\rho^{\prime}, \psi^{\prime}, X^{\prime}-X\right)$ in a frame attached to the sensor, centered on $\mathbf{x}_{\perp}$ and such that $\mathbf{d} \cdot \mathbf{d}^{\prime}=R^{2} c \psi^{\prime}$, we also have :

$$
\begin{aligned}
\frac{1}{r} & =\frac{1}{\left(l_{X}^{2}+2 R^{2}\left(1-c \psi^{\prime}\right)\right)^{1 / 2}}=\frac{\left(1 /\left|l_{X}\right|\right)}{\left(1+2 \xi_{X}^{2}\left(1-c \psi^{\prime}\right)\right)^{1 / 2}} \\
& =\frac{1}{\left|l_{X}\right|}\left(1-\xi_{X}^{2}\left(1-c \psi^{\prime}\right)\right)+O\left(\xi_{X}^{4}\right) .
\end{aligned}
$$

This can rewritten as :

$$
\begin{aligned}
& \sum_{\beta=1}^{m} \int_{\mathcal{I}_{\beta}}\left(\frac{\partial \phi_{1}}{\partial n}\right) \frac{1}{r} d s=\sum_{\beta=1}^{m} \int_{\mathcal{L}_{\mathcal{I}_{\beta}}} \frac{R \mathbf{E}_{1}}{\left|l_{X}\right|} . \\
& \int_{0}^{2 \pi} \mathbf{n}\left(1-\xi_{X}^{2}+\xi_{X}^{2} c \psi^{\prime}+O\left(\xi_{X}^{4}\right)\right) d \psi^{\prime} d X^{\prime} .
\end{aligned}
$$

Moreover, since $\mathbf{n}=c \psi^{\prime} \mathbf{j}_{c}+s \psi^{\prime} \mathbf{k}_{c}$, we obtain :

$$
\begin{aligned}
\sum_{\beta=1}^{m} \int_{\mathcal{I}_{\beta}}\left(\frac{\partial \phi_{1}}{\partial n}\right) \frac{1}{r} d s & =\sum_{\beta=1}^{m} \int_{\mathcal{L}_{\mathcal{I}_{\beta}}} \mathbf{E}_{1} \cdot\left(\mathbf{0}+\mathbf{O}\left(\xi_{X}^{3}\right)\right) d X^{\prime} \\
& =0+O\left(\xi_{X}^{3}\right) .
\end{aligned}
$$

Therefore, if each $\mathcal{E}_{\alpha}$ is sufficiently far from the others, the term (89) has no influence on (28) except on a small strip neighboring the electrode $\mathcal{E}_{\alpha}$ which generates a contribution modeled by pushing the expansion of (28) at the next order as in Appendix D. Finally, due to the smallness of the electrodes $\mathcal{E}_{\alpha}$, at leading order the field $\phi_{1}$ is approximated as uniform on each of them, and so we have also for any $\mathrm{x} \in \mathcal{C}_{X_{\alpha}} \subset \mathcal{E}_{\alpha}$ :

$$
2 \pi \phi_{1}(\mathbf{x})=2 \pi \phi_{1}\left(\mathbf{x}_{c \alpha}\right)(1+O(R / r)),
$$

and :

$$
\begin{aligned}
& \sum_{\beta=0}^{m} \int_{\mathcal{E}_{\beta}} \phi_{1} \frac{\partial(1 / r)}{\partial n} d s= \\
& \sum_{\beta=0}^{m} \phi_{1}\left(\mathbf{x}_{c \beta}\right) \int_{\mathcal{E}_{\beta}} \frac{\partial(1 / r)}{\partial n} d s(1+O(R / r)) .
\end{aligned}
$$

Hence at the leading order, equation (28) can be approximated as follows :

$$
\begin{aligned}
& 2 \pi\left(-\phi_{1}\left(\mathbf{x}_{c \alpha}\right)\right) \simeq \sum_{\beta=0}^{m}\left[\int_{\mathcal{E}_{\beta}}\left(\frac{\partial \phi_{2}}{\partial n}\right) \frac{1}{r} d s\right]- \\
& \sum_{\beta=0}^{m}\left[\int_{\mathcal{E}_{\beta}} \frac{\partial(1 / r)}{\partial n} d s\left(-\phi_{1}\left(\mathbf{x}_{c \beta}\right)\right)\right] \\
& -\sum_{\beta=1}^{m} \int_{\mathcal{I}_{\beta}} \phi_{2} \frac{\partial(1 / r)}{\partial n} d s,
\end{aligned}
$$

where we recognize the same expression as (27), with $\phi_{2}$ replacing $\phi_{0}$ and for $\alpha=0,1, \cdots, m$, $-\phi_{1}\left(\mathbf{x}_{c \alpha}\right)$ replacing $U_{\alpha}$. Consequently, the calculation of $\bar{I}^{(2)}$ is similar to the calculation of $\bar{I}^{(0)}$. Also, at the leading order, the axial conductance matrix $\bar{C}^{(0)}$ in the absence of an object is once again sufficient to calculate the response of the sensor submitted to a potential $\phi_{1}$ since we obtain (50). 


\section{Appendix D : Proof of (55)}

Now let us consider the approximation of (28) at the next order. In this case, the values of the electric fields can be considered as uniform on each of the electrodes $\mathcal{E}_{\alpha}$. By prolonging these values to the narrow insulating rings which bound the electrodes, and contribute to (28) in a non-negligible way, we can rewrite $(28)$ for any $\mathbf{x} \in \mathcal{E}_{\alpha}$ :

$$
\begin{aligned}
& \sum_{\beta=\alpha-1}^{\alpha}\left[\int_{\mathcal{I}_{\beta}} \frac{\partial(1 / r)}{\partial n}\left(\mathbf{x}^{\prime}-\mathbf{x}_{c \beta}\right) d s\right] \cdot \mathbf{E}_{2}\left(\mathbf{x}_{c \beta}\right)+ \\
& \sum_{\beta=0}^{m}\left[\int_{\mathcal{E}_{\beta}} \frac{\mathbf{n}}{r} d s\right] \cdot \mathbf{E}_{2}\left(\mathbf{x}_{c \beta}\right) \\
& \simeq \sum_{\beta=0}^{m}\left[\int_{\mathcal{E}_{\beta}} \frac{\partial(1 / r)}{\partial n}\left(\mathbf{x}^{\prime}-\mathbf{x}_{c \beta}\right) d s+2 \pi\left(\mathbf{x}-\mathbf{x}_{c \beta}\right) \delta_{\alpha \beta}\right] \\
& . \mathbf{E}_{1}\left(\mathbf{x}_{c \beta}\right)+\sum_{\beta=\alpha-1}^{\alpha}\left[\int_{\mathcal{I}_{\beta}} \frac{\mathbf{n}}{r} d s\right] \cdot \mathbf{E}_{1}\left(\mathbf{x}_{c \beta}\right),
\end{aligned}
$$

that once written in one point of each of the $\mathcal{E}_{\alpha}$, can be put formally in the explicit linear form :

$$
\text { For } \alpha=0,1, \ldots m: \mathbf{E}_{2}\left(\mathbf{x}_{c \alpha}\right)=\sum_{\beta=0}^{m} \mathbf{S}_{\alpha \beta} \cdot \mathbf{E}_{1}\left(\mathbf{x}_{c \beta}\right),
$$

where the $\mathbf{S}_{\alpha \beta}$ define a set of second order tensors of $\mathbb{R}^{3} \otimes \mathbb{R}^{3}$ modeling the polarization of the sensor to the electric field reflected by the object. Now, following the same expansion as in Appendix C (with respect to $\xi_{X_{\alpha}}$ ), the mutual influences terms between different electrodes $\mathcal{E}_{\alpha}$ are negligible with respect to the self influence ones, and we have :

$$
\mathbf{E}_{2}\left(\mathbf{x}_{c \alpha}\right) \simeq \mathbf{S}_{\alpha \alpha} \cdot \mathbf{E}_{1}\left(\mathbf{x}_{c \alpha}\right),
$$

where due to the axisymmetry of the sensor, the tensors $\mathbf{S}_{\alpha \alpha}$ take the form :

$$
\mathbf{S}_{\alpha \alpha}=s_{\| \alpha}\left(\mathbf{i}_{c} \otimes \mathbf{i}_{c}\right)+s_{\perp \alpha}\left(\mathbf{j}_{c} \otimes \mathbf{j}_{c}\right)+s_{\perp \alpha}\left(\mathbf{k}_{c} \otimes \mathbf{k}_{c}\right)
$$

with $s_{\| \alpha}$ and $s_{\perp \alpha}$ two positive eigen values. Finally, invoking the definition (18) of $I^{(2)}$, we have for any $e_{k} \subset \mathcal{E}_{\alpha(k)}$ and with $\mathbf{n}=\mathbf{n}_{\|}+\mathbf{n}_{\perp}$ as in Appendix B :

$$
\begin{aligned}
& I_{k}^{(2)}=\gamma \int_{e_{k}}\left(\mathbf{S}_{\alpha(k) \alpha(k)} \cdot \mathbf{E}_{1}\right) \cdot \mathbf{n} d s \\
& =\gamma \int_{e_{k}} s_{\| \alpha(k)} \mathbf{E}_{1} \cdot \mathbf{n}_{\|} d s+\gamma \int_{e_{k}} s_{\perp \alpha(k)} \mathbf{E}_{1} \cdot \mathbf{n}_{\perp} d s .
\end{aligned}
$$

Now, it is obvious that the first contribution (from left to right) is axisymmetric while the second has a lateral symmetry. Thus, the first term contributes to $I_{a x}^{(2)}$ but in a negligible way with respect to the leading order term of Appendix C. On the other hand the second contribution has to be added to $I_{\text {lat }}^{(1)}$ to which it contributes at the same order. Hence, we will have in this case :

$$
\begin{aligned}
I_{l a t, k}=I_{l a t, k}^{(1)}+I_{l a t, k}^{(2)}= & \gamma \mathbf{E}_{1} \cdot \int_{e_{k}}\left(1+s_{\perp \alpha(k)}\right) \mathbf{n}_{\perp} d s \\
& =\left(1+s_{\perp \alpha(k)}\right) I_{l a t, k}^{(1)},
\end{aligned}
$$

or again in matrix form :

$$
I_{\text {lat }}=\left(1+S_{\perp}\right) I_{\text {lat }}^{(1)},
$$

which corresponds to (56) with $S_{\perp}=\operatorname{diag}\left(s_{\perp \alpha(k)}\right)$. 


\section{Acknowledgment}

The ANGELS project is funded by the European Commission, Information Society and Media, Future and Emerging Technologies (FET) contract number : 231845. The authors gratefully acknowledge Christine Chevallereau and Alexis Girin ${ }^{6}$ for kindly sharing their materials on observers.

6. are with the IRCcYN lab 


\section{Références}

[1] H. Lissmann and K. Machin, "The mechanism of object location in gymnarchus niloticus and similar fish," The Journal of Experimental Biology, no. 35, pp. 451-486, 1958.

[2] J. Jackson, Classical Electrodynamics. Wiley and Sons, 3rd. ed., 1998.

[3] B. Rasnow, "The effects of simple objects on the electric field of apteronotus," Journal of Comparative Physiology A, vol. 3, no. 178, pp. 397-411, 1996.

[4] M. MacIver, E. Fontaine, and J. Burdick, "Designing future underwater vehicles : principles and mechanisms of the weakly electric fish," IEEE Journal of Oceanic Engineering, pp. 651-659, 2004.

[5] M. Nelson, M. MacIver, and S. Coombs, "Modeling electrosensory and mechanosensory images during the predatory behavior of weakly electric fish," Brain, Behavior and Evolution, vol. 59, pp. 199-210, 2002.

[6] J. Solberg, K. Lynch, and M. MacIver, "Robotic electrolocation : Active underwater target localization," International Conference on Robotics and Automation, 2007.

[7] — - "Active electrolocation for underwater target localization," The International Journal of Robotics Research, vol. 27, no. 5, pp. 529-548, 2008.

[8] G. Baffet, F. Boyer, and P. Gossiaux, "Biomimetic localization using the electrolocation sense of the electric fish," in Robotics and Biomimetics ROBIO IEEE, 2008.

[9] B. Jawad, P. Gossiaux, F. Boyer, V. Lebastard, F. Gomez, N. Servagent, S. Bouvier, A. Girin, and M. Porez, "Sensor model for the navigation of underwater vehicles by the electric sense," in Robotics and Biomimetics ROBIO IEEE, 2010.

[10] V. Lebastard, C. Chevallereau, A. Amrouche, B. Jawad, A. Girin, F. Boyer, and P. Gossiaux, "Underwater robot navigation around a sphere using electrolocation sense and kalman filter," in IROS 2010 IEEE, 2010.

[11] M. Porez, V. Lebastard, A. J. Ijspeert, and F. Boyer, "Multy-physics model of an electric fishlike robot : Numerical aspects anbd application to obstacle avoidance," in 2011 IEEE/RSJ Int. Conf. on Intelligent Robots and Systems, (San Francisco, CA), (to appear in), 2011.

[12] J. Happel and H. Brenner, Low Reynolds number hydrodynamics. Prentice Hall, 1965.

[13] M. Morse and H. Feschbach, Mathematical methods in physics, 1953.

[14] Y. Liu, Fast multipole boundary element method. Cambridge university press, 2009.

[15] L. Gaul, M. Kögl, and M. Wagner, Boundary element methods for engineers and scientists. Springer-Verlag, 2003.

[16] A. Caputi, R. Budelli, and C. Bell, "The electric image in weakly electric fish : physical images of resistive objects in gnathonemus petersii," Journal of Experimental Biology, vol. 201, no. 14, pp. 2115-2128, 1998.

[17] G. V. D. Emde, S. Schwarz, L. Gomez, R. Budelli, and K. Grant, "Electric fish measure distance in the dark," Letters to Nature, Nature, vol. 395, pp. 890-894, 1998.

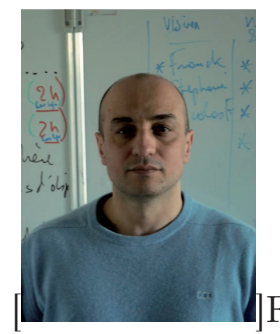

Frédéric BOYER was born in France in 1967. He received a Diploma in Mechanical Engineering from the Institut Nationale Polytechnique de Grenoble, Grenoble, France 
in 1991, a Master of Research degree in Mechanics from the University of Grenoble in 1991 and a Ph.D. degree in Robotics from the University of Paris VI, Paris, France in 1994. He is currently a Professor at the Department of Automatic Control, Ecole des Mines de Nantes (France), where he works with the Robotics Team, Institut de Recherche en Communication et Cybernétique de Nantes (IRCCyN). His current research interests include structural dynamics, geometric mechanics, and bio-robotics. Prof. Boyer was awarded the Monpetit prize of the Academy of Science of Paris in 2007 for his work in dynamics.

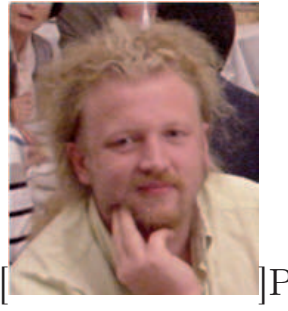

Pol Bernard Gossiaux Pol Bernard Gossiaux was born in Belgium in 1967. He completed his Ph.D. in theoretical nuclear physics at the university of Liege in 1993. From 1993 till 1997, he occupied 3 postdoctoral positions at the university of Nantes, at the Max Planck Institut for Nuclear Physics of Heidelberg and at the Michigan State University. He is currently full professor at the Ecole des Mines de Nantes, working at the SUBATECH laboratory in the high energy theory group. His main research interests are the physics of heavy ions collisions and of the quark-gluon plasma, and physics modelling in general. He has taught electromagnetism for 10 years.

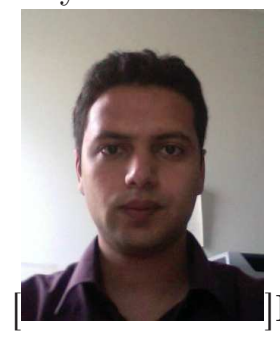

Brahim JAWAD Brahim Jawad received a Master of Research degree in physics from the university of Rouen, France, in 2007. He is currently working toward a Ph.D. degree in the modeling of electrolocation in biorobotics with the Ecole des Mines de Nantes, Institut de Recherche en Communication et Cybernétique de Nantes.

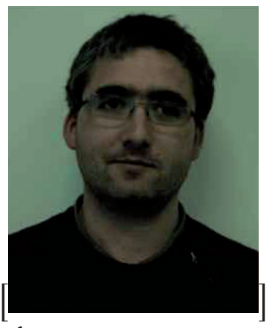

Vincent Lebastard Vincent Lebastard was born in France in 1977. He joined the École Normale Supérieure de Cachan, France, in 2001, and received an aggregation from the ministry of the Education in Electrical Engineering in 2002. He received a Ph.D degree from the University of Nantes, France, in 2007. Currently, he is an assistant professor at Ecole des Mines de Nantes and member of IRCCyN. His research interests include biorobotics and nonlinear control and observation.

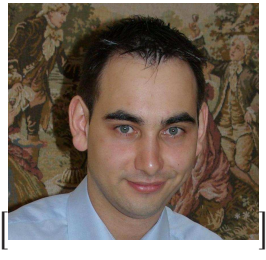

Mathieu Porez born in France in 1980. He obtained a University Degree of Technology in Mechanics and Industrial Engineering from Université de Rennes (France) in 2000. He obtained a Master of Technology in Material forming and a Master of Science in 
Material and Fluid Mechanics from University of Lorient (France) in 2003. He obtained a Ph.D of Robotics from University of Nantes in 2007. From February 2009 to August 2010, he has been a postdoctoral researcher at the BioRob Laboratory of EPFL (Switzerland). He is currently assistant professor at Ecole des Mines de Nantes, working at IRCCyN laboratory in the Robotic group. His research topics include Bio-Robotics and Fluid Mechanics. 